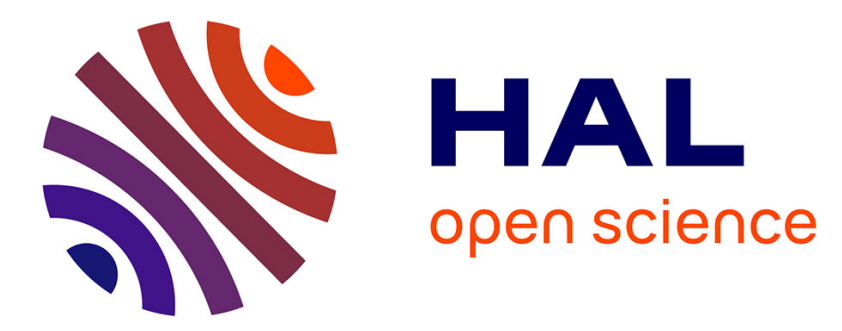

\title{
Impact of dynamic modelling of the flame subgrid scale wrinkling in large-Eddy simulation of light-round in an annular combustor
}

Stefano Puggelli, Denis Veynante, Ronan Vicquelin

\section{- To cite this version:}

Stefano Puggelli, Denis Veynante, Ronan Vicquelin. Impact of dynamic modelling of the flame subgrid scale wrinkling in large-Eddy simulation of light-round in an annular combustor. Combustion and Flame, 2021, 230, pp.111416. 10.1016/j.combustflame.2021.111416 . hal-03394365

\section{HAL Id: hal-03394365 https://hal.science/hal-03394365}

Submitted on 22 Oct 2021

HAL is a multi-disciplinary open access archive for the deposit and dissemination of scientific research documents, whether they are published or not. The documents may come from teaching and research institutions in France or abroad, or from public or private research centers.
L'archive ouverte pluridisciplinaire HAL, est destinée au dépôt et à la diffusion de documents scientifiques de niveau recherche, publiés ou non, émanant des établissements d'enseignement et de recherche français ou étrangers, des laboratoires publics ou privés. 


\title{
Impact of dynamic modelling of the flame subgrid scale wrinkling in Large-Eddy Simulation of light-round in an annular combustor
}

\author{
Stefano Puggelli ${ }^{a}$, Denis Veynante ${ }^{\mathrm{a}}$, Ronan Vicquelin ${ }^{\mathrm{a}, *}$ \\ ${ }^{a}$ Laboratoire EM2C, CNRS, CentraleSupélec, Université Paris-Saclay, 8 - 10 Rue Joliot Curie, 91192, Gif-sur-Yvette Cedex, France
}

\begin{abstract}
Ignition of annular gas turbine combustors, also called light-round, is a challenging problem for aeronautic reliability and safety. Large Eddy Simulation (LES), based on massively parallel computations, has been already assessed as a reliable tool to analyse and predict such a phenomenon. The present study focuses on the effect of the flame subgrid scale wrinkling factor entering the combustion model. Large Eddy Simulations of the annular MICCA-Spray laboratory-scale chamber are performed with constant and dynamic strategies for the wrinkling parameter and are compared against experimental data. A bi-modal distribution of the model exponent during light-round is obtained with a different behaviour between the flames stabilized around each injector, and the flame fronts propagating in a circumferential direction. The impact of such locally defined and time-dependent evolution is analysed by studying several quantities associated to the flame propagation mechanisms. It is shown that the dynamic procedure entails a reduction of the wrinkling factor that is not balanced by an increase of the resolved flame surface. After considering the exhaust of burnt gases that affects the ignition in such a semi-confined system, the dynamic formulation leads to a moderate slowing down of the flame. Surprisingly, the sounder dynamic model does not yield better predictions of the experimental data, revealing the need to address other modelling improvements in the future. However, this does not temper the need for a dynamic combustion model in numerical simulation of light-round.
\end{abstract}

Keywords: Dynamic modelling; ignition; light-round; annular combustor; turbulent flame wrinkling

\section{Introduction}

Among issues that have to be faced during the design of modern aero-engines, the ignition of the combustor is a critical and central one. A reliable and safe ignition of the full chamber has to be guaranteed over a wide range of operating points, and this can be particularly difficult under altitude relight conditions. A deep insight into flame initiation and propagation is, therefore, crucial to estimate the ignitability of the combustor chambers. Several studies in technical literature have been focused on characterizing the fundamental ignition mechanisms, and four successive steps are usually recognized [1, 2]: (A) the kernel initiation by a spark plug; (B) the kernel growth, which, in case of successful ignition, is followed by (C) the flame propagation, which ends with the generation of a stable turbulent flame. In multipleinjector configurations, such as aero-engines, the generated flame propagates from one sector to the next.

${ }^{*}$ Corresponding author: ronan.vicquelin@ @entralesupelec.fr
The propagation continues up to the full-ignition of the chamber. This last step (D) is usually identified as lightround.

The first three steps have been investigated from both experimental and numerical perspectives [3-6], mainly at ambient conditions. The stage (D) has received more and more attention over the last years. Both linear and annular geometrical configurations have been studied to achieve an insight into flame propagation in multiburner setups. Barré et al. [7] performed an extensive characterization of flame propagation based on experiments carried out on a linear array comprising five injectors, employing both gaseous [7] and liquid fuels [8]. The study was mainly focused on the impact of the inter-injector distance both on the flame propagation pattern and velocity. However, the annular configuration, typically adopted in aero-engines, shows specific phenomena that cannot be investigated on linear arrays (e.g. the distance interaction of the two flame fronts, which propagate in the chamber in opposite directions, or the merging phase that concludes the light-round). 
Therefore, experimental facilities have been designed to collect information about light-round in annular systems. Bourgouin et al. [9] were first to provide data on the flame propagation in a laboratory-scale multiple injector annular system, named MICCA, fueled with a premixed air-propane mixture. Recently, Prieur et al. $[10,11]$ included also liquid injection: the flame propagation process studied on the MICCA-Spray configuration exhibits the same stages reported in gaseous conditions [9], although the light-round duration and then the flame propagation speed strongly differ. Machover and Mastorakos $[12,13]$ proposed as well ignition experiments in a multi-burner annular combustor fed with an air-methane mixture, focusing the attention on the impact of a non-premixed injection and of the swirler spacing. Similarly, in [14], experimental tests have been performed at Zhejiang University on an annular chamber fuelled with a lean air/propane mixture, collecting information on the effects of the ignition mode on the flame propagation pattern and on the resulting lightround time.

To complement the information provided by these experimental campaigns, Large Eddy Simulation (LES) has been employed. Boileau et al. [15] were the first able to investigate the full ignition of a helicopter engine through LES. More recently, massively parallel LES were employed in the premixed gaseous injection case $[16,17]$ and with liquid fuel $[18,19]$ to analyze the MICCA-Spray chamber. Similarly, Xia et al. [20] carried out LES on the annular chamber studied in [14] employing Automatic Mesh Refinement (AMR) and a Perfectly Stirred Reactor (PSR) approach for chemistry description.

Generally, computations have been able to correctly retrieve the most significant features of light-round, such as the flame propagation pattern and the light-round duration.

However, several modelling hypotheses were included in the various numerical setups that deserve further investigations. Among them, most numerical simulations [15-19] relied on an algebraic model formulation for the flame subgrid-scale wrinkling factor. This assumption may, however, not be adapted for transient and timedependent phenomena such as ignition and flame propagation [21].

The main objective of the present work is to assess the effect of an improved model for the subgrid-scale flame wrinkling, based on a dynamic formulation. The impact of a different expression of the flame subgrid scale wrinkling has never been analysed in the context of lightround and it represents the specific aim of this work. An alternative to algebraic models can be dynamic clo- sures, which adjust the subgrid-scale wrinkling factor based on the time-dependent and locally defined resolved flow conditions. A filtering procedure is carried out on the resolved fields with a test filter scale larger than the LES filter. A Germano-like equation [22] is then applied to compute the wrinkling factor. Several applications are listed hereinafter and show the maturity of this modelling strategy. Im et al. [23] and Knudsen et al. [24] performed the application of dynamic models in the G-equation context, selecting as validation case the F3 jet flame investigated by Chen et al. [25]. In the Thickened Flame (TFLES) context, Wang et al. [26] applied such procedure on the same jet flame, whereas, more recently, Volpiani et al. [27] studied with a similar numerical setup the PRECCINSTA burner [28, 29]. The dynamic approach has been also coupled with the flame surface density model of Boger et al. [30] in [31] and with tabulated chemistry in $[32,33]$ for the Tecflam [34] and for the Cambridge stratified burner [35] respectively. All these works were aimed at assessing the effect of a dynamic closure for the flame subgrid scale wrinkling on different turbulent premixed flames and at showing the higher accuracy that can be obtained with respect to algebraic models.

Several works have been also focused on the application of the dynamic approach in the context of explosions: Ibrahim et al. [36] and Gubba et al. [37] applied the Dynamic Flame Surface Density (DFSD) model, proposed by Knikker et al. [38], to simulate a turbulent premixed flame through obstacles. A similar work was carried by Volpiani et al. [39] in the context of TFLES, investigating the deflagrating flame studied experimentally by Masri et al. [40]. These works confirmed that dynamic modelling, since it avoids tuning of the wrinkling model, presents a significant advantage with respect to algebraic models also in the context of explosions.

Therefore, a significant amount of works have been already focused on the validation of dynamic strategies in different type of configurations. However, the application of such approach in the framework of gas turbine ignition and, more specifically, for the light-round phase is missing.

The goal of the present work is therefore to quantify and understand the effects of dynamic modelling on the light-round of the MICCA-Spray chamber. Two new computations with algebraic and dynamic subgrid-scale approaches are considered. While all the other aspects of the numerical setup are fixed, only the turbulent combustion model is varied. Results are compared with the available experimental data. A thorough comparison of the two calculations is proposed: volume-integrated and surface-averaged quantities are first analysed; a macro- 
scopic balance of burnt gases is then presented and this allows for relating the flame propagation speed for lightround to the dynamic closure. This a posteriori study hence fully connects the impact of the modified wrinkling parameter to the achieved absolute flame speed of the flame during the light-round phase simulation.

The article is structured as follows. Sections 2-3 are devoted to the description of the MICCA-Spray system and of the numerical setup. Results of calculations corresponding to light-round with or without the dynamic formulation for the flame subgrid-scale wrinkling factor are discussed and analysed in Sec. 4. In Sec. 4.1, the attention is focused on the qualitative description of the effects of the dynamic closure on the wrinkling parameter, while Secs. 4.2-4.3 are aimed at detailing its impact on the light-round duration and the turbulent flame wrinkling, respectively.

\section{Experimental Configuration}

The experimental setup and technical instrumentation are briefly described in this section. More details may be found in [10, 11]. MICCA-Spray is a laboratory scale annular combustion chamber representative in an idealized way of aero-engines combustors (see Fig. 1). It is composed of sixteen swirled injectors equally distributed in a tangential pattern. Each injector of diameter $d_{i n j}=8 \mathrm{~mm}$ is equipped with a simplex atomizer, which feeds liquid n-heptane as sketched in Fig. 1(a). The chamber is enclosed by two quartz windows with diameters $D_{\text {in }}=149 \mathrm{~mm}$ and $D_{\text {out }}=201 \mathrm{~mm}$ that allow optical access to the flame to investigate the flame propagation. A spark igniter, located at sector $S_{0}$ (see Fig. 1(b)) triggers the ignition process. Eight photomultipliers (PM) are placed in front of each injector on the $H^{-}$halve (see Fig. 1(b)) to estimate the flame inter-sector times. The sign of each halve of the chamber is defined with respect to the rotation of the flow in the swirlers that is shown in Fig. 1(b) for injector $S_{1}$. The burnt gases are exhausted into an open atmosphere. The test conditions investigated in the present article are briefly summarised in Tab. 1 .

\section{Numerical Modelling}

The main characteristics of the numerical setup are given in this section. Several numerical details were already discussed in $[18,19]$. Hence, the relevant aspects are summarized in what follows, and then the attention is mainly focused on the dynamic approach for subgridscale wrinkling, which is the objective of the present study.

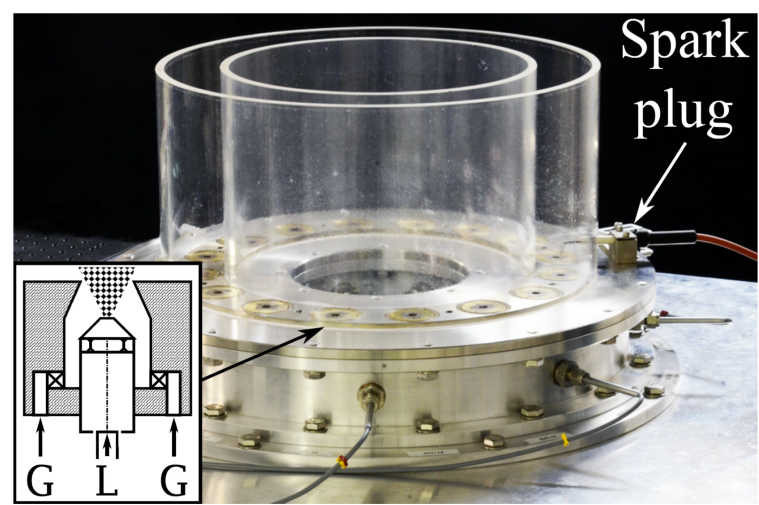

(a)

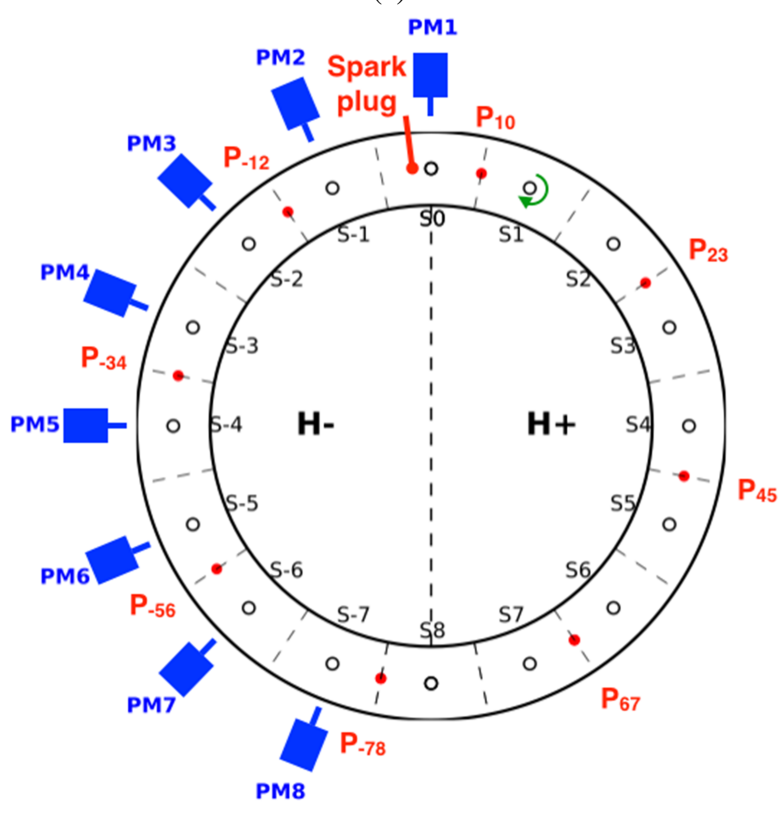

(b)

Figure 1: Experimental MICCA-Spray combustion chamber (a) and sketch view of the available diagnostics (b). The swirler is sketched on the bottom-left corner showing gas (G) and liquid (L) injections. Taken from [41]

\subsection{Numerical configuration}

All simulations are carried out with the LES solver AVBP, jointly developed by Cerfacs and IFPEN. This code solves the 3D Navier-Stokes equations for compressible and reactive flows [42]. The subgrid-scale turbulent contributions are represented with the Wall Adapting Local Eddy (WALE) model, as proposed by Nicoud and Ducros [43].

The liquid phase is modeled through a mono-disperse Eulerian-Eulerian approach. This simplified methodol- 


\begin{tabular}{cc}
\hline \hline Inlet pressure [bar] & 1 \\
Inlet gas temperature $[\mathrm{K}]$ & 300 \\
Global equivalence ratio $[-]$ & 0.89 \\
Total air flow rate $[\mathrm{g} / \mathrm{s}]$ & 30.10 \\
Fuel flow rate per injector $[\mathrm{g} / \mathrm{s}]$ & 0.111 \\
Nominal thermal power $[\mathrm{kW}]$ & 79.3 \\
\hline \hline
\end{tabular}

Table 1: Operating conditions of the MICCA-Spray chamber.

ogy has been shown to provide a good compromise between accuracy and CPU cost [18, 19], and the same numerical setup is retained in the present calculations. Transport equations for the mass fraction, droplet number density, momentum and enthalpy of the liquid phase are solved jointly with the gas phase balance equations [44]. Models for droplet motion, evaporation and heat transfer are required in order to represent the interactions between the two phases properly. Drag contribution is introduced in the liquid momentum equation based on the hypothesis of spherical droplets. The evaporation model assumes a uniform temperature over the radius for the droplets, and the evaporation rate is calculated through the formulation of Abramzon and Sirignano [45]. The effects of the subgrid contributions on the liquid-phase are neglected. A two-step TaylorGalerkin weighted residual central distribution numerical scheme (TGCC), third-order in time and space [46], is used for both liquid and gaseous phases.

The combustion model, describing the flame propagation and its interaction with turbulence, is the TFLES, as proposed by Colin et al. [47]. It is worth mentioning that, despite the two-phase characteristics of MICCASpray, Lancien [48] showed that the propagating flame is mainly characterized by a premixed burning mode, while the non-premixed zones are limited to the near injection regions. Therefore, the application of TFLES in the present configuration is proposed as a convincing choice. The two-steps mechanism for n-heptane derived in [49] is applied for the chemical description. It should be pointed out that in the framework of ignition, a more detailed description of chemistry should be preferred. In particular, reduction techniques, based on Articially Reduced Chemistry (ARC) [50] or virtual chemistry [51], can be employed since they include some radical species that are important in the first stages of the flame development. However, in the present study, the attention is focused on the light-round process and not specifically on the flame initiation. In this context, the most significant aspect from the chemistry point of view is being able to reproduce the evolution of the laminar flame speed over a wide range of equivalence ratio. This is already ensured by the employed two-steps scheme, which is also characterized by a reduced CPU cost with respect to ARC and virtual chemistry.

The LES mesh cannot resolve the total flame wrinkling, and one must rely on models to recover the unresolved contributions. This issue is still the topic of ongoing research, and the major employed hypothesis to model the wrinkling factor is that an equilibrium is reached between the turbulent motion and the flame wrinkling at the subgrid-scale level [52]. Even if satisfactory results have been obtained with this assumption, it has been shown that it may fail under certain conditions [21, 39, 53, 54]. Therefore, two different formulations of the wrinkling factor are compared hereinafter:

(A) Algebraic static formulation: Charlette et al. [52] developed an algebraic formulation for the wrinkling factor $\left(\Xi_{\Delta}\right)$ assuming equilibrium between the turbulent motion and the flame wrinkling. It was then slightly modified in [26] to the following expression:

$$
\Xi_{\Delta}=\left(1+\min \left[\left(\frac{\Delta}{\delta_{l}}-1\right), \Gamma_{\Delta}\left(\frac{\Delta}{\delta_{l}}, \frac{u_{\Delta}^{\prime}}{S_{l}}, R e_{\Delta}\right) \frac{u_{\Delta}^{\prime}}{S_{l}}\right]\right)^{\beta}
$$

where $R e_{\Delta}=u^{\prime}{ }_{\Delta} \Delta / v$ and $u^{\prime}{ }_{\Delta}$ are the Reynolds number at subgrid-scale and the corresponding velocity fluctuation. The latter quantity, in the present study, has been estimated from the rotational part of the resolved velocity field, as suggested in [47]. The fresh gas kinematic viscosity is $\nu$, and $\beta$ is a user-defined model parameter. A value of $\beta_{\text {Charlette }}=0.5$ was suggested in [52], but it can be adjusted based on the configuration under investigation. Direct numerical simulations proved that Eq. (1) is often saturated [55], i.e. the term $\left[\left(\Delta / \delta_{l}\right)-1\right]$ controls the actual value of the wrinkling factor. In this case, Eq. (1) reduces to the following simplified form:

$$
\Xi_{\Delta}=\left(\frac{\Delta}{\delta_{l}}\right)^{\beta}
$$

Equation (2), if $\beta$ is assumed independent on scales and if $0<\beta<1$ [52], represents a fractal model with a flame surface of dimension $D=\beta+2$ and an inner cut-off scale corresponding to the laminar flame thickness $\delta_{l}$. Hence, the assumption of $\beta_{\text {Charlette }}=0.5$ over the entire flame corresponds to a constant fractal dimension and, consequently, to a uniform wrinkling over the flame front (if $\Delta / \delta_{l}$ 
is constant). This is not a satisfactory hypothesis in transient configurations or in complex technical applications, where $\Xi_{\Delta}$ should be expressed as a function of time and space.

(B) Dynamic formulation: Instead of imposing a fixed value of the wrinkling parameter, the $\beta$ coefficient can be dynamically computed $[21,39,56]$. In particular, it can be determined by equating the flame surfaces calculated at a filtered and testfiltered scales and employing a "Germano-like" equation:

$$
\left\langle\widehat{\Xi_{\Delta}|\nabla \tilde{c}|}\right\rangle=\left\langle\Xi_{\gamma \Delta}|\nabla \hat{\tilde{c}}|\right\rangle
$$

where $\tilde{c}$ is the progress variable, estimated in this work from $\mathrm{CO}$ and $\mathrm{CO}_{2}$ mass fractions. The hat symbol represents a test-filtering operation over the filter size $\hat{\Delta}$. The effective filter size $\gamma \Delta$ can be calculated as a combination of the Gaussian filters of size $\Delta$ and $\hat{\Delta}$ with $\gamma=\sqrt{1+(\hat{\Delta} / \Delta)^{2}}$. The $\langle\cdot\rangle$ symbol indicates the averaging operation carried out on the flame surfaces: both global and local formulations have been proposed in [21, 27]. In this work, the local procedure, where the averaging is performed over a filter size $\Delta_{a v g}$, has been retained. Following the work of Volpiani et al. [21], the averaging procedure has been replaced by a Gaussian filter easier to implement on unstructured grids.

Starting from Eq. (4) and combining it with Eq. (2), the following expression of the wrinkling parameter $\beta$ can be retrieved:

$$
\beta=\frac{\log (\langle\widehat{\nabla \tilde{c} \mid}\rangle /\langle|\nabla \hat{\tilde{c}}|\rangle)}{\log (\gamma)}
$$

This formulation has been validated on different conditions going from jet flames up to swirled configurations [21, 27] obtaining a satisfactory comparison with experimental data.

In this work, a recent update of dynamic modelling proposed by Mouriaux et al. [54] has been employed. In particular, such a proposal was aimed at solving overshoots in the $\beta$-parameter that occur when the flame fronts interact at the test-filter scale. Indeed, in this case, looking to Eq. (4), while $\Sigma_{1}=\widehat{|\nabla \tilde{c}|}$ preserves a continuous and smooth evolution, the flame surface density $\Sigma_{2}=|\nabla \hat{\tilde{c}}|$ may become zero, leading to nonphysical values of the $\beta$ parameter and of the wrinkling factor [54]. Therefore, Mouriaux et al. [54] developed a correction
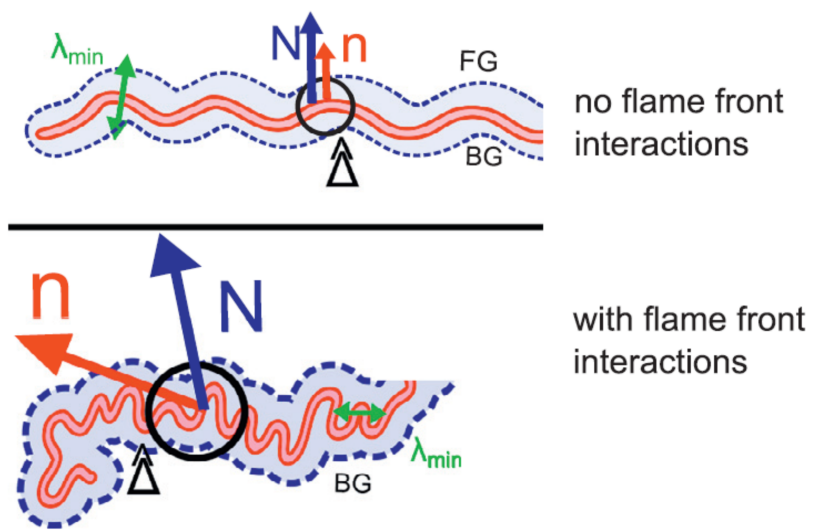

Figure 2: Sketch of the correction for cusps interaction developed in Mouriaux et al. [54]. Image taken from [54]. The red lines represent the flame fronts at the combustion filter size $\Delta$, while the blue surfaces are the respective estimations at the test-filter size $\hat{\Delta}$. The arrows $\mathbf{n}$ and $\mathbf{N}$ are respectively the normal to the resolved flame front at filter and test-filter scales.

for the flame surface density at test-filter scale that is sketched in Fig. 2. In order to detect a possible interaction at test-filter scale, the normal to the resolved $(N=-\nabla \tilde{c} /|\nabla \tilde{c}|)$ and to the filtered flame fronts $(n=-\nabla \hat{\tilde{c}} /|\nabla \hat{\tilde{c}}|)$ are computed. If the flame front is not too wrinkled (see Fig. 2), there are not interactions at test-filter scale and the vectors $\mathbf{n}$ and $\mathbf{N}$ are collinear. In this case, the cusps flag $\psi$ is set to zero. Instead, when the fronts interact at scale $\hat{\Delta}$, $\mathbf{n}$ and $\mathbf{N}$ have different directions and the cusps flag is imposed $\psi=1$. In the present work, the flames have been considered as interacting at testfilter scale if $\mathbf{N} \cdot \mathbf{n}<1-\epsilon$, where $\epsilon=0.05$. In case of interactions at test-filter scale, $\Sigma_{2}$ is corrected with $\Sigma_{3}=\mathbf{N} \widehat{\cdot \mathbf{n} \mid} \nabla \tilde{c} \mid$ that is not zero in presence of interactions between flame fronts. The following corrected expression for the flame surface density at test-filter scale is finally employed:

$$
\Sigma_{2}{ }^{c o r r}=(1-\hat{\psi}) \Sigma_{2}+\hat{\psi} \Sigma_{3}
$$

where the flag $\psi$ has been test-filtered $(\hat{\psi})$ since the presence of flame front interactions induce highvalues of the wrinkling factor over a region of characteristic size $\hat{\Delta}$. Ultimately, $\beta$ takes the following final form:

$$
\beta=\frac{\log \left(\langle\widehat{|\nabla \tilde{c}|}\rangle /\left\langle\Sigma_{2}{ }^{\text {corr }}\right\rangle\right)}{\log (\gamma)}
$$

In [54], a smooth evolution of $\beta$ and of the testfiltered flame surface was obtained with Eq. (6) in 


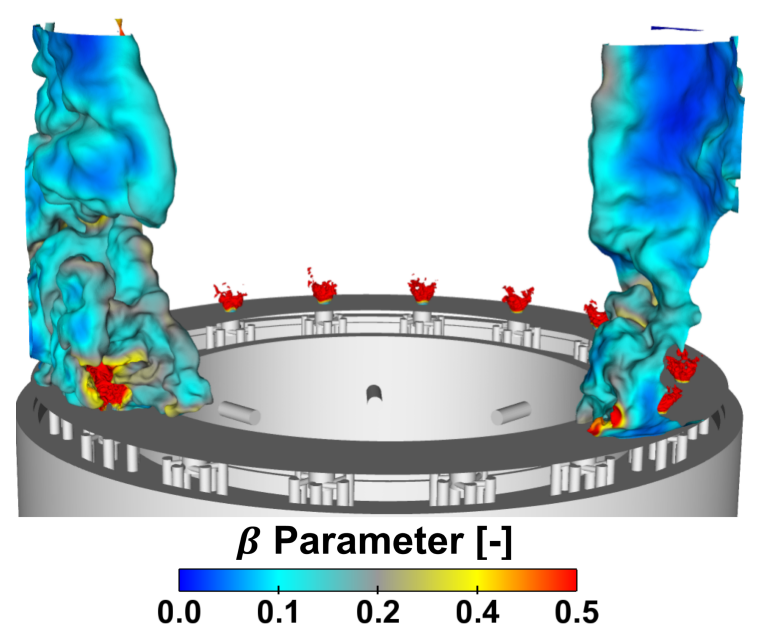

(a)

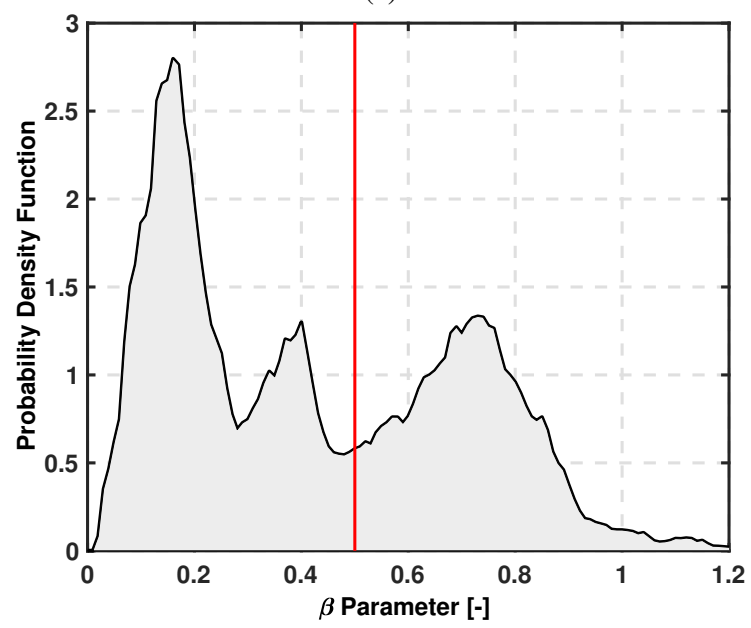

(b)

Figure 3: A-priori evaluation of dynamic closure in the light-round of MICCA-Spray. Iso-surface of $\tilde{c}=0.5$ coloured with the wrinkling parameter (a) and the corresponding $\operatorname{PDF}(\beta)$ over the $\tilde{c}$ iso-surface (b). Red vertical line points out the threshold value $\beta_{\text {Charlette }}=0.5$.

presence of $1 \mathrm{D}$ interacting flame fronts. The model was then applied to the spark ignition of an internal combustion engine [54].

Coherently with [26], once $\beta$ is computed thanks to Eq. (6), the wrinkling factor is then calculated based on Eq. (1). This procedure avoids the estimation of the subgrid-scale velocity fluctuation at filter and test-filter scales. Furthermore, in this manner, with respect to the MICCA-Spray calculation employing Eq. (1) with $\beta_{\text {Charlette }}=0.5$, it is possible to evaluate just the impact of the local and time-varying formulation of the wrinkling parameter on the flame propagation.

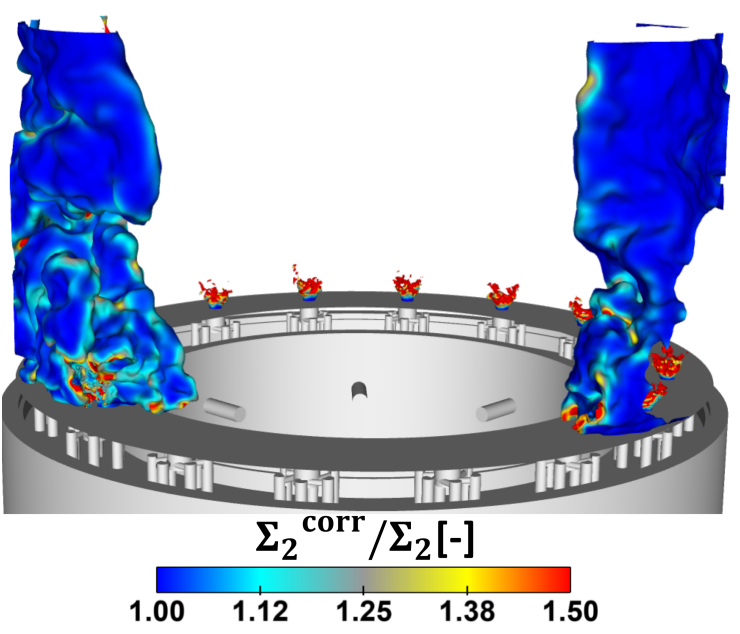

Figure 4: A-priori evaluation of dynamic closure in the light-round of MICCA-Spray. Iso-surface of $\tilde{c}=0.5$ coloured with the ratio $\Sigma_{2}{ }^{\text {corr }} / \Sigma_{2}$

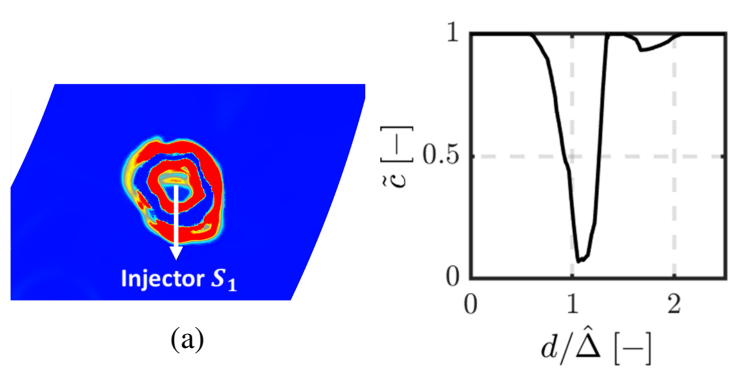

(b)

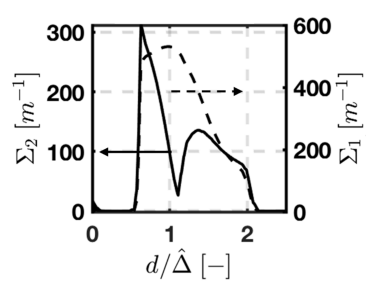

(c)

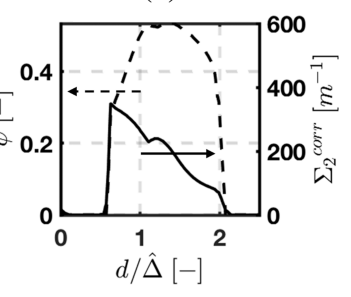

(d)
Figure 5: Evolution of some characteristic quantities of the dynamic model of Mouriaux et al. [54] over a normal cut to the flame front. Fig. 5(a) shows the position of the analysed flame front through the heat release contour plot and the position of the line cut (white arrow). Fig. 5(b) reports the evolution of the progress variable over such line, Fig. 5(c) the one of $\Sigma_{1}$ with dashed line and $\Sigma_{2}=|\nabla \hat{\tilde{c}}|$ with continuous line, Fig. 5(d) the one of the $\psi$-sensor as dashed line and $\Sigma_{2}{ }^{\text {corr }}$ as continuous line.

The importance of dynamic modelling in the context of flame propagation for MICCA-Spray can be assessed right away. In particular, starting from the data published in $[18,19]$ with the formulation of Charlette et al. [52] for the wrinkling factor, Fig. 3 shows the 


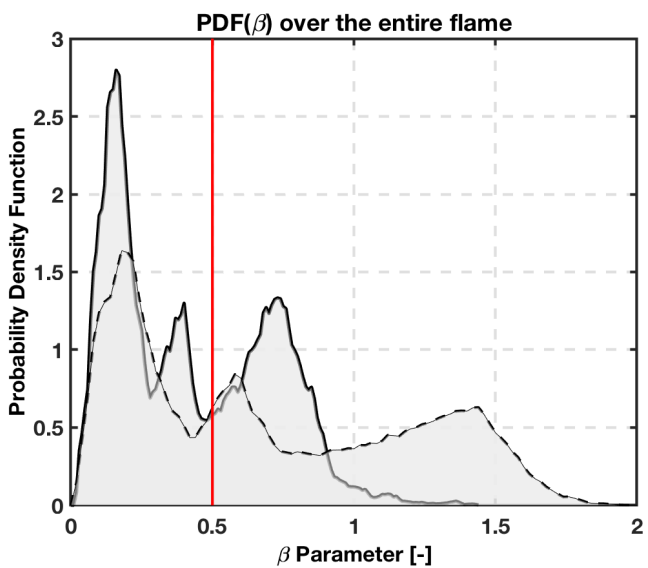

(a)

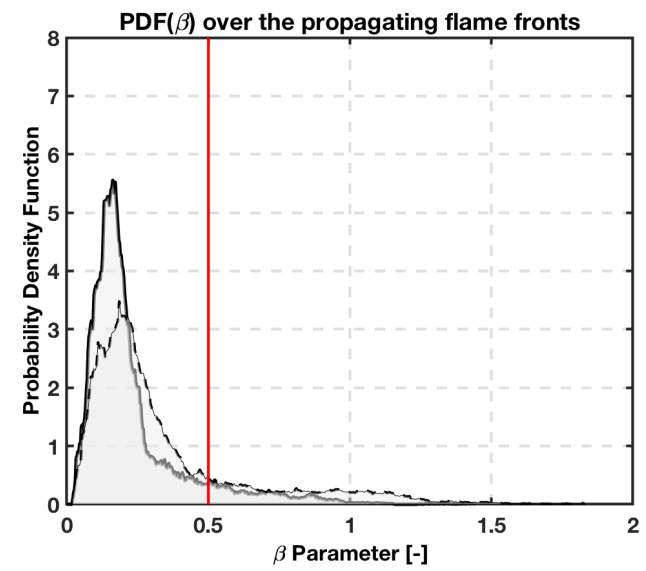

(b)

Figure 6: A-priori evaluation of dynamic closure in the light-round of MICCA-Spray. $\operatorname{PDFs}(\beta)$, extracted on the iso-surface $\tilde{c}=0.5$, over the entire flame (a) and on the propagating flame fronts (b), obtained from the model of Mouriaux et al. [54] (continuous black line) and without the correction for interacting flame fronts (dashed black line). The propagating flame fronts have been extracted through a geometrical clipping. Red vertical line points out the threshold value $\beta_{\text {Charlette }}=0.5$.

results obtained from an a-priori evaluation of the dynamic procedure. The chosen snapshot corresponds to a time-frame when the flame has already ignited the firsthalf of the chamber. The investigated test point corresponds to Tab. 1. The flame is visualized by an isosurface of progress variable $\tilde{c}=0.5$ coloured with the $\beta$-parameter, ranging from $\beta=0$ in blue to $\beta>0.5$ in red (see Fig. 3(a)). The corresponding probability density function $P D F(\beta)$ over the $\tilde{c}$ iso-surface is reported in Fig. 3(b).

Two main behaviors can be identified: values of $\beta$ lower than $\beta_{\text {Charlette }}$ in zones where the flame propagates cir- cumferentially, and higher values at the flames attached around each injector. The corresponding PDF assumes an approximative bi-modal shape that reflects this dual evolution.

It is worth also assessing at this stage the a-priori impact of the cusps correction (see Eq. (5)) provided by the model of Mouriaux et al. [54]. Figure 4 shows the iso-surface of $\tilde{c}=0.5$ coloured with the ratio $\Sigma_{2}{ }^{\text {corr }} / \Sigma_{2}$ in order to point out the zones where the cusps correction is having the larger effect. The same time-frame of Fig. 3 has been chosen here. In the flame propagation regions, the corrector is barely active, and local front interactions are identified just where the flame is more wrinkled. Instead, it is triggered on the flames stabilized in each sector. These zones are characterized by much higher turbulence levels and stronger curvatures of the flame fronts, thus inducing the activation of the $\psi$-sensor. This aspect is better detailed in Fig. 5(a), where the flame stabilized at the injector $S_{1}$ (see Fig. 1) is displayed thanks to the the heat release rate field obtained on a normal plane to the chamber axis at a distance $X=5 \mathrm{~mm}$ from the combustor backplane. Due to the M-shape of the flame, two peaks of heat release are predicted by the numerical simulations, corresponding to the shear layers between fresh air and the burnt gases in the inner and outer recirculation zones. Figures 5(b)5(d) show several profiles to better understand the impact of the cusps correction in this zone. These profiles are cuts through the flame front. The chosen cut is represented by a white arrow on the heat release rate contour in Fig. 5(a). The abscissa is the distance along the arrow scaled by the local value of the test filter $(\hat{\Delta})$. The evolution of $\tilde{c}$ in Fig. 5(b) points out the presence of a gradient of the progress variable within the testfilter size and therefore the presence of interacting flame fronts. While $\Sigma_{1}=\widehat{|\nabla \tilde{c}|}$, shown with dashed black line in Fig. 5(c), has non-zero values within the flame front, $\Sigma_{2}=|\nabla \hat{\tilde{c}}|$, plotted as continuous black curve, tends to zero, leading to an ill-posed computation of the wrinkling parameter (i.e. $|\widehat{\nabla \tilde{c}}| \gg|\nabla \hat{\tilde{c}}|$ in Eq. (4)). This problem is overcome by the modified formulation proposed in [54]. The $\psi$-sensor is activated, as shown in Fig. 5(d) as a dashed black line, and, based on Eq. (5), this leads to a consistent evolution of $\Sigma_{2}{ }^{\text {corr }}$ (black continuous line in Fig. 5(d)) and therefore of the $\beta$-parameter.

The effect of this correction on the instantaneous $\operatorname{PDF}(\beta)$ is shown in Fig. 6. The a-priori values of the wrinkling parameter obtained with the model of Mouriaux et al. [54] are compared with the ones calculated through the dynamic procedure of Eq. (4). In Fig. 6(a), the PDFs are compared over the entire flame, show- 
ing the significant impact of the cusps corrector in removing the non-physical overshoots of $\beta$ generated on the single-sector flames. However, if the attention is just focused on the flame fronts that are propagating in a circumferential direction during light-round (see Fig. 6(b)), the effect of the cusps corrector is much more moderate, and it does not change substantially the distribution of the $\beta$-parameter.

Utlimately, this a-priori output clearly shows that the wrinkling parameter is not uniform and that it varies in space and time based on local flow conditions. The correction proposed in [54] and employed in the present study seems to be beneficial to avoid non-physical overshoots in the $\beta$ distribution. The range of values of the wrinkling parameter is wide, and the threshold $\beta_{\text {Charlette }}$ is not representative of any moment of the corresponding $\beta$-distribution. Therefore, the employed dynamic procedure can have a non-negligible effect on the flame propagation speed and on the resulting light-round time.

\subsection{Numerical setup}

As in previous investigations [16-19], the numerical domain represents the exact geometry of MICCA-Spray (see Fig. 1) including the air plenum, the swirlers, and the chamber. A large volume is also included to evacuate the burnt gases and mimic the outlet atmosphere. Standard Navier-Stokes characteristic boundary conditions [57] are imposed at the inlet, outlet, and lateral walls. Air mass-flow rates have been prescribed on the eight tubes that feed the plenum coherently with the experimental values reported in Tab. 1. Atmospheric pressure has been fixed at the outlet. Moreover, considering that experimentally the chamber walls were preheated up to reaching a steady-state temperature before triggering the ignition, consistently with previous works on MICCA [16-19], all the walls are assumed adiabatic. The liquid spray injection conditions are those derived in Lancien et al. [18], where an optimum droplet size is computed for the mono-disperse injection of MICCA-Spray. The methodology proposed in [18] relies on computing multiple LES of the single sector corresponding to MICCA-Spray to assemble a continuous response surface based on Polynomial Chaos Expansion using as uncertain parameter the injection diameter. The obtained meta-model is combined with an error criterion, based on the two-phase laminar flame speed, to compute the optimal spray injection diameter. The reader interested in this topic is addressed to [18] where several details on this procedure are provided. The optimal diameter computed in this manner is coupled with the profiles of droplets' velocities and particle number density experimentally measured at $2 \mathrm{~mm}$ from the injector exit to complete the set of spray boundary conditions.

Two calculations have been carried out in the present work, one by using the algebraic static formulation of [52] (i.e. Eq. (1)) and one with dynamic modelling (i.e. Eq. (6)). In the latter case, the test-filter size has been set equal to $\hat{\Delta}=2 \Delta$, whereas the averaging size has been fixed to $\Delta_{a v g}=2.7 \Delta$, consistently with previous works [21, 39]. In [21], it has been shown that the output of the dynamic model marginally depends on these parameters. This is confirmed later on during the discussion of the results. The effective combustion filter size $\Delta$ is unknown in TFLES since the thickening procedure is not equivalent to filtering the flame front in a LES context. Consistently with [21], an equivalent TFLES filter size has therefore been defined as $\Delta=\alpha F \delta_{l}$ where $\alpha=1.4$, and $F$ and $\delta_{l}$ are respectively the thickening factor and the laminar flame thickness, here defined on temperature gradient. The values of the $\beta$ parameter are updated every 750 iterations of the main solver. With this setup and by limiting the filtering operation geometrically within the combustion chamber (thus avoiding the plenum and the outer atmosphere) and in the flame region, the computational over-cost linked to dynamic model is $+20 \%$. In Appendix A, an assessment of the effect on the $\beta$-parameter of limiting the filtering operation geometrically and in the flame zone is reported.

The initial conditions to start the ignition process correspond to the ones employed in Lancien et al. [18] for the same test point here investigated. In [18], given the geometrical periodicity of the MICCA-Spray chamber, one-eighth of the combustor has been simulated with tangential periodic boundary conditions to compute the fully developed non-reacting two-phase mixture. The convergence has been verified to ensure the experimental value of the equivalence ratio on the whole chamber. Once converged, the solution has been replicated in the azimuthal direction to fill the whole chamber geometry. The full 360deg solution, obtained in this manner, has been then used in the present work for the reactive calculations as initial condition. The ignition is initiated by introducing a sphere of hot burnt gases at injector $S_{0}$ to mimic the presence of the spark plug with a radius $r_{\text {sphere }}=5 \mathrm{~mm}$ and a temperature profile obtained from a n-heptane-air 1D laminar solution. The detailed investigation of the first instants of flame initiation is beyond the goals of the present work. However, the characteristics of the initial flame kernel might influence the temporal development of the flame, especially when using a dynamic model to estimate the unresolved flame wrinkling from the resolved one. The 360deg MICCA-Spray mesh counts 288 million elements and 50 million nodes. 


\section{Results}

This section reports the numerical results obtained on MICCA-Spray by employing the algebraic formulation of Charlette et al. [52] and the dynamic closure. Experimental data are also shown serving as a reference. A thorough analysis of the distribution of the wrinkling parameter on the flame is carried out to evidence that the assumption of $\beta_{\text {Charlette }}=0.5$ is not valid and that a local formulation has to be preferred. Then, the effect of $\beta$ on the flame propagation speed and on the inter-sector flame passage time is discussed to present the net effect of the employed dynamic procedure. Finally, the investigation is focused on understanding the impact of the dynamic closure on the wrinkling factor that is a crucial element to define the flame propagation speed during light-round.

\subsection{A-posteriori analysis of the $\beta$ field}

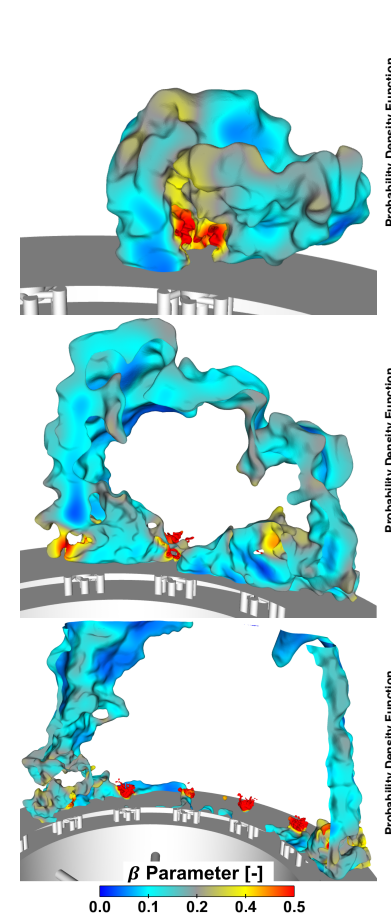

(a)
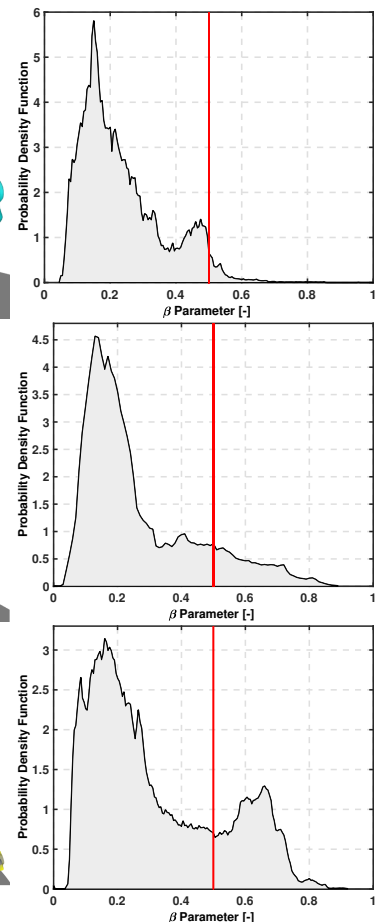

(b)

Figure 7: Light-round sequence of MICCA-Spray with dynamic modelling at different instants : $8.3 \mathrm{~ms}, 15 \mathrm{~ms}, 23 \mathrm{~ms}$, coloured with the wrinkling parameter (a) and the corresponding $\operatorname{PDF}(\beta)$ over the $\tilde{c}$ iso-surface (b). Red vertical line points out the threshold value $\beta_{\text {Charlette }}=0.5$.

Figures 7- 8 show the qualitative evolution of the flame shapes obtained with the dynamic closure. The
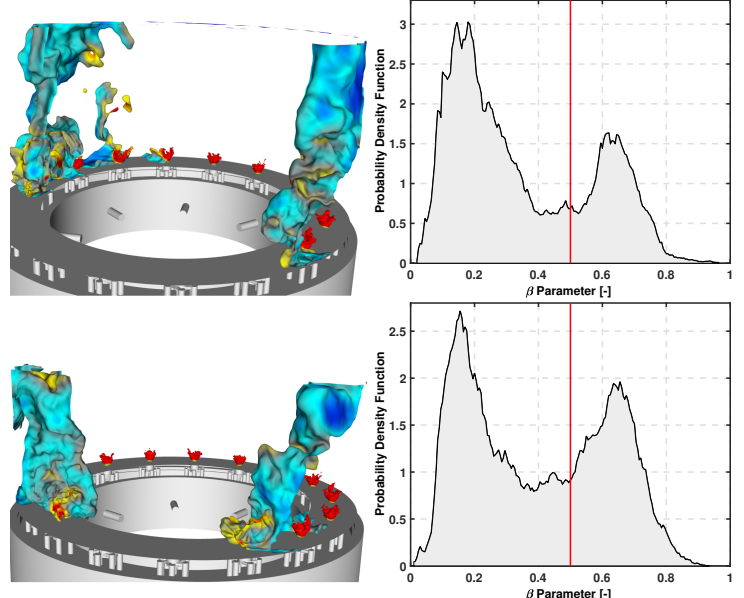

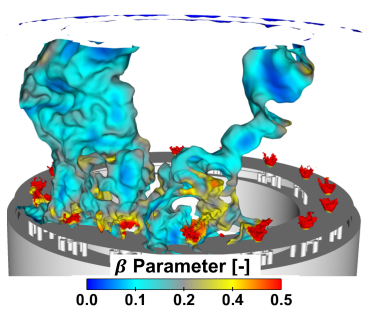

(a)

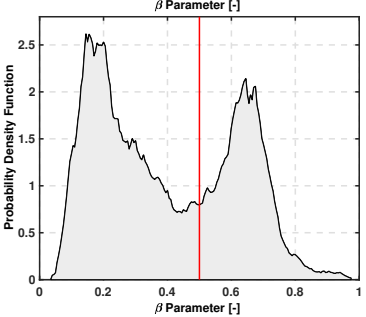

(b)
Figure 8: Light-round sequence of MICCA-Spray with dynamic modelling at different instants : $38 \mathrm{~ms}, 46 \mathrm{~ms}$ and $62 \mathrm{~ms}$, coloured with the wrinkling parameter (a) and the corresponding $\operatorname{PDF}(\beta)$ over the $\tilde{c}$ iso-surface (b). Red vertical line points out the threshold value $\beta_{\text {Charlette }}=0.5$.

flame front is evidenced with an iso-surface of progress variable $\tilde{c}=0.5$ coloured with the wrinkling parameter, ranging from $\beta=0$ in blue to $\beta>0.5$ in red. The color scale has been saturated on purpose to highlight the regions where $\beta$ is lower than $\beta_{\text {Charlette. For }}$ each time-frame, the corresponding $\operatorname{PDF}(\beta)$ over the progress variable iso-surface is displayed. All the PDFs are cell-based, so resolution dependent. In Appendix A, while discussing the filtering operation, an assessment about the effect of the value of the progress variable chosen to extract the $P D F(\beta)$ is reported to show that the discussion carried out hereinafter has a general meaning and it is not impacted by choice of $\tilde{c}=0.5$. The time for each snapshot is measured, starting from the instant when the ignition has been triggered.

After the initial propagation of the kernel, named hereinafter as phase-I ( $t=8.3 \mathrm{~ms}$ in Fig. 7), an arch-like shape is generated with a consequent propagation in a circumferential direction. At this stage, the flame moves towards sectors $S_{1}$ and $S_{-1}$ and the chamber exit. This corresponds to the phase-II of light-round $(t=15 \mathrm{~ms}$ in 
Fig. 7). It is clearly apparent that the trend anticipated in Fig. 3 is confirmed: in the zones where the flame fronts are expanding in a radial direction, the wrinkling parameter is smaller than $\beta_{\text {Charlette }}$. The opposite trend is instead predicted for the flames attached around each injector: the color scale is completely saturated (i.e., $\beta>0.5$ ).

After reaching the exit of the chamber, at $t=23 \mathrm{~ms}$, two distinct fronts, which are almost vertical and propagate in a tangential direction, are generated. This instant determines the beginning of phase-III. The two-zones evolution of $\beta$ is confirmed and, indeed, the $P D F(\beta)$ assumes a clear bi-modal distribution. Progressing in time (i.e. $t=38 \mathrm{~ms}, 46 \mathrm{~ms}$ in Fig. 8), the same trend is retrieved up to arriving to the merging of the flame fronts (i.e. $t=62 \mathrm{~ms}$ in Fig. 8) and to the full ignition of the chamber. At this stage, which corresponds to the phase- $I V$, the flames stabilized around each injector are characterized by similar distributions of $\beta$. These phases have been observed in all the previous works on MICCA-Spray [10, 11, 18, 19] both experimentally and numerically. However, now the additional information that the wrinkling parameter is not constant over the flame front is available thanks to the dynamic procedure. This represents the novel knowledge on the numerical modelling of light-round that is obtained in this work with respect to the state of the art.

To further deepen this point, the aforementioned dual distribution of $\beta$ is further analysed:

- Flame propagation regions: The evolution of the wrinkling parameter in the zones where the flame front is propagating can be analysed by studying the flame leading point (LP), which is the most advanced flame point in the azimuthal direction. This element represents a key quantity to better understand the mechanisms involved in the flame propagation and, as shown in [19], it can provide meaningful information about the whole lightround process. In Fig. 9, the wrinkling parameter at the leading point is plotted for the two halves of the chamber. It should be pointed out that the curves behave differently. Indeed, as shown in Figs. 7-8, the flame does not propagate symmetrically since the $\mathrm{H}^{+}$-front is more advanced in a circumferential direction than the other one. The geometry is not symmetrical due to the orientation of the swirlers, and this can explain the different behavior of the leading point for the two halves. In particular, it is expected that the flame front should go faster when the flow at the outer part of the swirler is aligned with the propagation direction.
Nevertheless, consistently with Figs. 7-8, while the flame is propagating, $\beta$ is small with an average value around $\bar{\beta}_{L P}=0.25$. Therefore, the employed dynamic closure entails an averaged reduction of the wrinkling parameter with respect to Charlette et al. [52] about 50\%, which is a remarkable drop compared to previous works on the same subject [21, 39].

Moreover, by closely looking at Figs. 7-8, this local behaviour is not limited to the leading point, but it is representative of all the regions where the flame is propagating. This can be further highlighted by computing the volume integral of the wrinkling parameter over the flame front $\left(\beta_{a v}=\right.$ $\int_{V_{c c}} \beta|\nabla \tilde{c}| d V / \int_{V_{c c}}|\nabla \tilde{c}| d V$, where $V_{c c}$ is the volume of the combustion chamber), which is plotted in Fig. 10. Consistently with the leading point analysis, even if this averaged value is taking into account also the flames stabilized around each injector, the major part of the flame front is characterized by low values of the wrinkling parameter, with an average value $\bar{\beta}_{a v}=0.22$. The dependency of this result on the test-filter size has been also assessed: assuming a fractal behaviour of the flame surface and feeding the conservation of the total flame surface (i.e. $\Xi_{\Delta} S_{r}=\Xi_{\gamma \Delta} \hat{S}_{r}$ ) with Eq. (2), the following relation between the resolved flame surface at filter $\left(S_{r}\right)$ and test-filter $\left(\hat{S}_{r}\right)$ scales can be obtained:

$$
\frac{\hat{S}_{r}}{S_{r}}=\frac{\Xi_{\Delta}}{\Xi_{\gamma \Delta}}=\left(\frac{\gamma \Delta}{\Delta}\right)^{-\beta}=\gamma^{-\beta}
$$

Hence, the ratio $\hat{S}_{r} / S_{r}$ should be a straight line of slope $-\beta$ when plotted as a function of the effective test filter size $\gamma$ in a log-scale. This is shown in Fig. 11, where the test-filter size is varied from $1.5 \Delta$ to $7 \Delta$ starting from the instantaneous snapshot shown in Fig. 3. As in [21, 39], the filtered flame surface $\left(\hat{S}_{r}\right)$ has been computed from the norm of the filtered progress variable gradient over the whole chamber volume (i.e $\hat{S}_{r}=\int_{V_{c c}}|\nabla \hat{\tilde{c}}| d V$ ). The scaling of the flame surfaces as a function of the filter size is confirmed. The slope of the curve gives $\beta=0.25$. This proves the robustness of the dynamic approach since a very good agreement has been obtained between theory (Eq. (7) and Fig. 11) and numerical results (Fig. 10). As in previous works [21, 39], it is also confirmed that the model parameter does not depend significantly on the test filter width.

- Flames stabilized around each injector: Pro- 


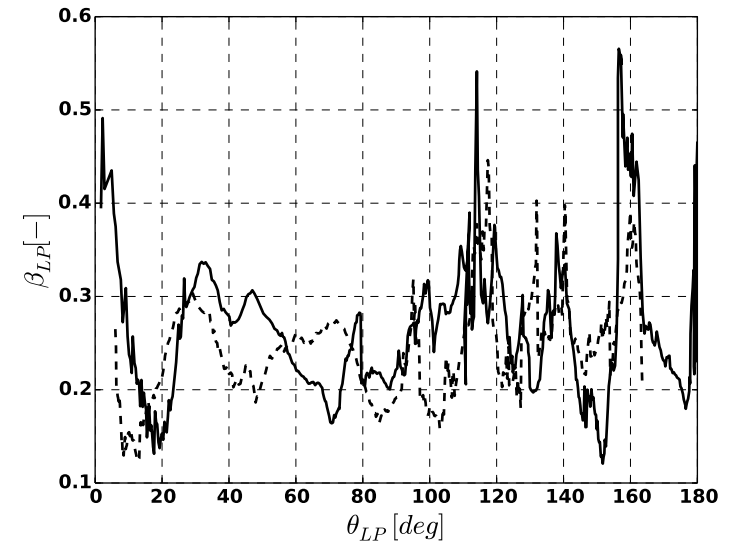

Figure 9: Evolution of the wrinkling parameter at the flame leading point for the $H^{+}$(solid black line) and $H^{-}$(dashed black line) halves of the chamber. See the right-side of Fig. 1 for the definition of the sign of two halves with respect to the rotation of the flow in the swirler.

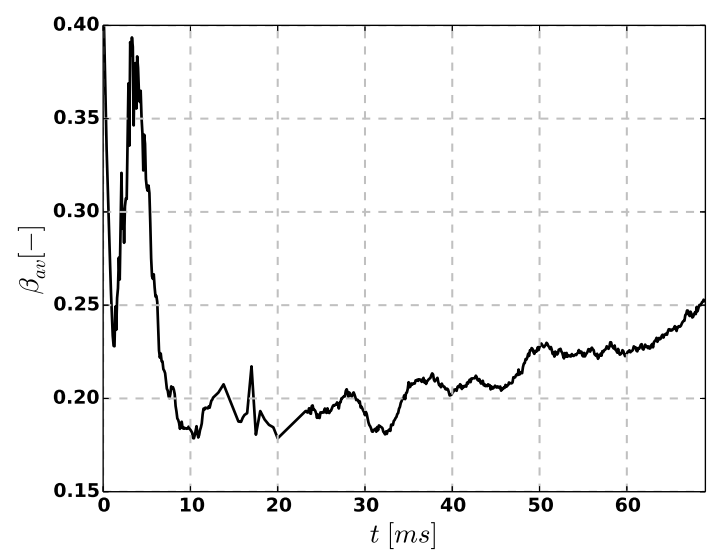

Figure 10: Evolution of the volume integral of the wrinkling parameter over the flame front.

gressing in the light-round, the flames stabilized around each injector assume similar features that are detailed in Fig. 12. In Fig. 12(a), the flame attached at the injector $S_{1}$ is shown by displaying the iso-surface of $\tilde{c}=0.5$ coloured with the wrinkling parameter. In Fig. 12(b), the corresponding $\operatorname{PDF}(\beta)$ is plotted. Here, the evolution is completely different to what was reported above for the leading point: a M-shape flame is stabilized at the injector exit with large regions where $\beta$ is higher than $\beta_{\text {Charlette. }}$. The wrinkling parameter is small in the initial flame region and increases downstream, while the flame is wrinkled by the turbulence. This

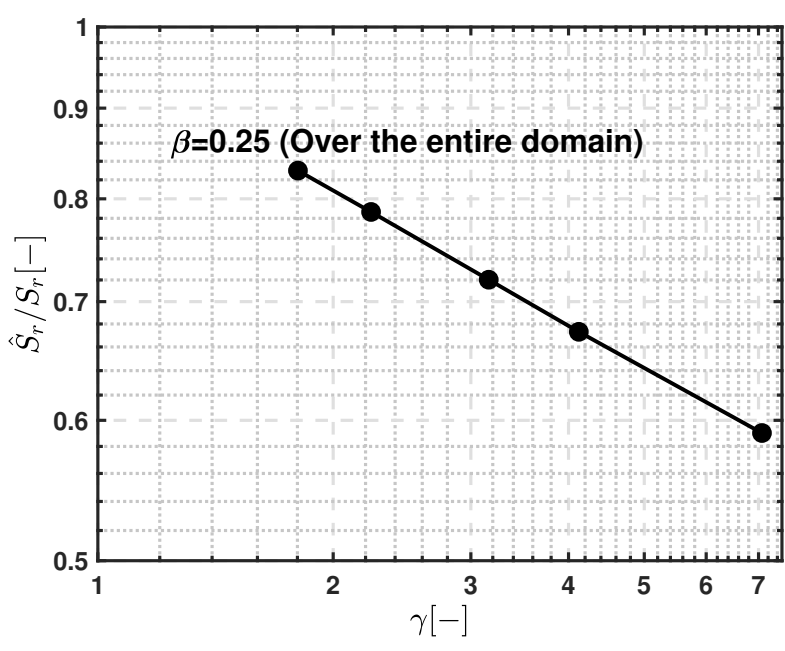

Figure 11: Normalized filter flame surface, $\hat{S}_{r} / S_{r}$, as a function of $\gamma$ (Eq. (7)) in log-scale for different values of the test-filter size. Surfaces have been computed from the instantaneous snapshot shown in Fig. 3. The shown $\beta$ value corresponds to the mean slope.

evolution is in agreement with previous findings for similar configurations [21, 39]. The corresponding PDF takes a wide range with a modal value shifted around $\beta_{\text {modal }}=0.65$.

Hence, this qualitative description clearly highlights the importance of a locally defined and time-dependent evaluation of the wrinkling parameter in light-round calculations. Indeed, neither the standard assumption of $\beta_{\text {Charlette }}=0.5$ nor the averaging of Eq. (6) over the volume of the whole chamber would lead to a consistent representation. A dual distribution of $\beta$ has to be taken into account: the flame wrinkling is led by mechanisms that depend on the local characteristics of the flow-field that vary significantly between the injector regions and the propagating flame fronts.

In the next section, the effect of such dynamic description on the flame propagation speed is investigated. This will be carried out by using volume integral quantities and a novel estimation of the flame propagation speed in the context of light-round for the present semi-confined configuration.

\subsection{Impact of $\beta$ on the flame propagation}

The effect of the dynamic model can be analysed by studying the evolution of the heat release rate (HRR) as integrated over the volume of the chamber $\left(H R R^{T O T}=\right.$ $\left.\int_{V_{c c}} H R R d V_{c c}\right)$. Figure 13 compares $H R R^{T O T}$ as obtained with the dynamic $\beta$-formulation and employing $\beta_{\text {Charlette }}=0.5$. Vertical lines have been added for 


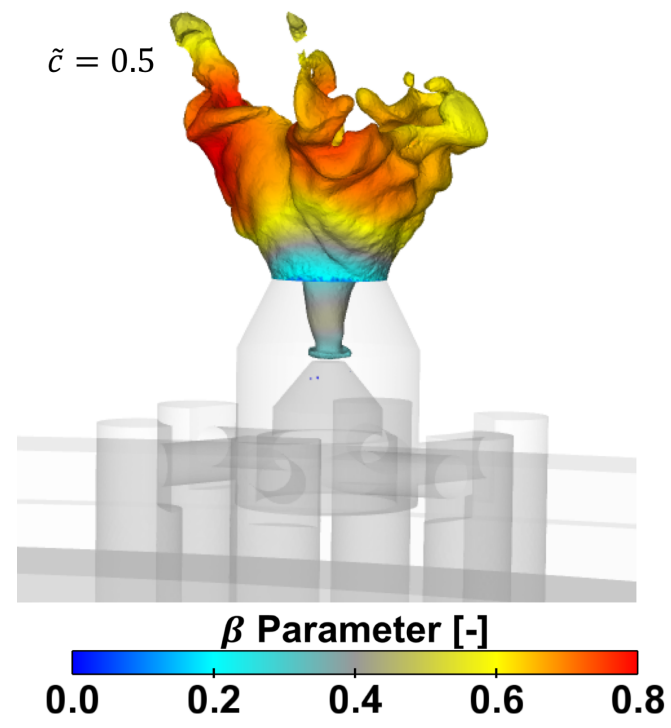

(a)

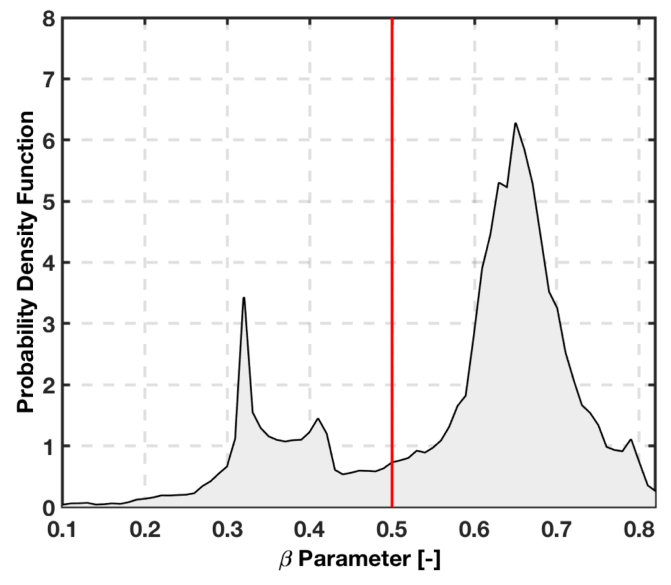

(b)

Figure 12: Iso-surface of $\tilde{c}=0.5$ coloured with the wrinkling parameter $\beta$ for the flame stabilized around injector $S_{1}$ (a) and corresponding $P D F(\beta)(b)$. Red vertical line points out the threshold value $\beta_{\text {Charlette }}=0.5$.

both calculations to show the duration of the numerical phases identified in Sec. 4.1 while describing the light-round process. Consistently with previous works [16-19], after triggering the ignition, during phase-I, an initial stagnation of the heat release profile is predicted by numerical simulations, then followed by a progressive increase. This leads to the beginning of phase-II, when, with an arc-like shape of the flame front, the calculations present a steep increase due to the expansion of burnt gases. Then, once the flame reaches the chamber exit, the transition between phase-II and phase-III takes place and $H R R^{T O T}$ keeps increasing with a lower slope up to arriving to the merging of the two flame fronts, which marks the start of phase- $I V$. At this stage, a sudden reduction of the heat-release is predicted by the two computations that corresponds to the evacuation of burnt gases through the outlet and to the end of the ignition of the initial fresh gases in the combustion chamber. This reduction has been simulated in the constant $\beta$ case up to reaching a drop of $50 \%$ in heat release. Instead, in the dynamic case, the computed time has been limited to a lowering of $10 \%$ to save computational resources. Trend lines have been then included in the graph to point out the tendency of the two curves. As reported in previous works [16-19], the heat release will progressively reduce up to reaching a plateau value representative of steady-state combustion in the chamber.

It should be pointed out that the dynamic formulations predicts already in phase-I a lower slope with respect to the static formulation. This most likely has to be linked to the methodology employed in the present work to trigger the ignition, which is based on imposing a laminar spherical kernel of burnt gases (see Sec. 3.2). However, by doing so, Eq. (4) is not valid if the imposed flame kernel is smaller than the filter size since the flame front is not fully resolved. In this work, the spherical laminar flame front should be large enough to immediately apply the dynamic procedure. Nevertheless, in this manner, an underestimation of the subgridscale wrinkling is induced since the resolved flame kernel is not yet wrinkled during the initial development phase. This problem is not present instead for the static model of Charlette et al. [52]. Two solutions can be considered: as done in Mouriaux et al. [54], a kernel already grown and wrinkled can be employed as initial solution for the dynamic simulation. However, in this manner, the predicted sub-grid wrinkling would be controlled by the initial solution. A more general way would consist in the development of a dedicated ignition model, able to describe the flame dynamics from the spark up to time when the dynamic methodology can be applied. However, this goes beyond the scope of the present study considering that the interest is not focused on these initial stages of flame development, but mostly on the light-round phase. Therefore, to have a consistent comparison between dynamic and static formulations, the same ignition procedure has been kept, but this might justify the smoother evolution of $H R R^{T O T}$ obtained in phase-I with the dynamic methodology.

Beyond this initial phase, even if the time evolution in the two cases is qualitatively the same, the slope predicted in the dynamic case is generally lower and the 
constant $\beta$ calculation is characterized by higher values of the heat release all along the light-round. This suggests a reduction of the flame speed with dynamic $\beta$.

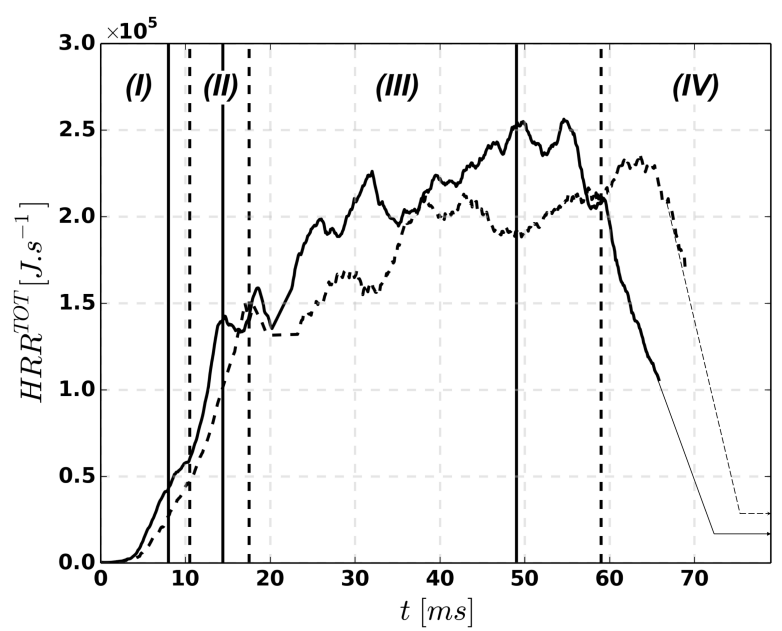

Figure 13: Time evolution of the integral of rate of heat release obtained with a constant $\beta_{\text {Charlette }}=0.5$ (solid black line) and employing a dynamic formulation (dashed black line). Trend lines are added at the end of light-round with solid and dashed thin lines respectively for the constant and dynamic $\beta$ cases. Vertical lines highlight the beginning and the end of the numerical phases of light-round, identified by Roman numbers, for the constant (solid black line) and dynamic (dashed black line) computations.

To have a deeper insight on this latter point, the flame absolute flame speed is introduced. From a theoretical perspective, the absolute velocity of a flame front element, $S_{a}$, represents its speed with respect to a fixed reference system. It can be computed as:

$$
S_{a}=\mathbf{w} \cdot \mathbf{n}=\frac{1}{|\nabla \tilde{c}|} \frac{\partial \tilde{c}}{\partial t}
$$

with $\mathbf{w}$ the absolute velocity (in a fixed referential system) of a $\tilde{c}$ iso-surface and $\mathbf{n}$ the vector normal to the flame front. $S_{a}$ can be directly related to the volume of burnt gases, $V_{b}$. An expression can be then derived (see Appendix B) for the average of the absolute velocity over the resolved flame surface $\left(S_{a, r e s}\right)$ :

$$
S_{a, r e s}=\frac{\int_{V_{c c}} S_{a}|\nabla c| d V}{A_{\text {res }}}=\frac{1}{A_{\text {res }}} \frac{d V_{b}}{d t}
$$

where $A_{\text {res }}$ is the resolved flame surface computed as $A_{\text {res }}=\int_{V_{c c}}|\nabla \tilde{c}| d V$. The corresponding evolution for the constant and dynamic cases is plotted in Fig. 14(a). In Fig. 14(b), the associated time behaviour of $V_{b}(t)$ is also plotted for both computations. After the triggering of ignition, $S_{a, r e s}$ increases with a sharp gradient during

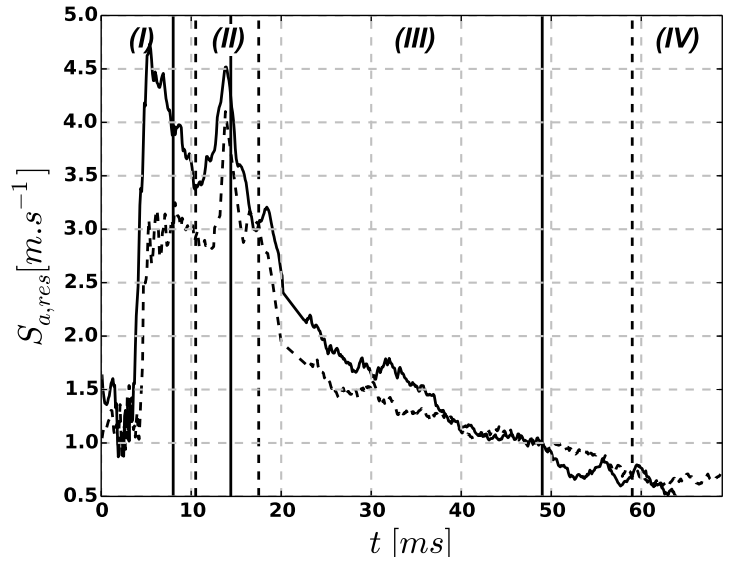

(a)

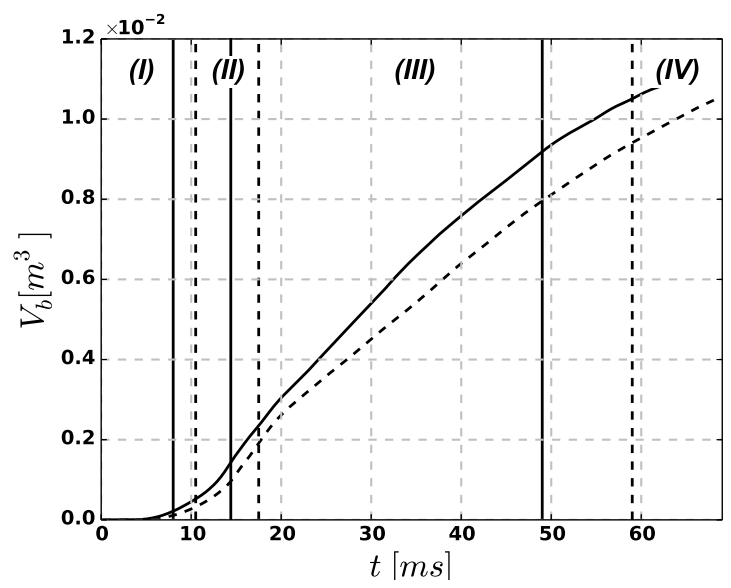

(b)

Figure 14: Time evolution of absolute velocity over the resolved flame surface computed with Eq. (25) (a) and corresponding development of the volume of burnt gases (b). Solid black lines correspond to the constant $\beta$ case and dashed curves to the dynamic formulation. Vertical lines highlight the beginning and the end of the numerical phases of light-round, identified by Roman numbers, for the constant (solid black line) and dynamic (dashed black line) computations.

phase-I. This acceleration of the flame results from the expansion of burnt gases that generates a flow field that transport the flame front at a high velocity as already observed in [16-19]. Then, while the flame is propagating with an arch-like shape, $S_{a \text {,res }}$ reaches a plateau for few milliseconds around $t=10 \mathrm{~ms}$. Finally, during phase-III, it decreases progressively up to the merging. Albeit the qualitative evolution is similar in the two simulations, the modification in the formulation of the wrinkling parameter leads to lower $S_{a, r e s}$. The same trend can be pointed out also by looking at the time evo- 
lution of $V_{b}$, where the values obtained with $\beta_{\text {Charlette }}$ are higher than in the dynamic case all along the lightround. This is the direct effect of the dynamic closure: the reduction in $\beta$ described in the above section entails a lowering of the propagation speed and therefore an expected augmentation of the light-round time.

However, $S_{a, r e s}$ is the average of the absolute flame speed over all the flame elements, regardless of their orientation. This velocity is not representative of the actual mean propagation speed of the flame during the light-round process.

As an alternative, in phase-III, when the fronts are propagating in a circumferential direction roughly as two vertical planes (see $t=23 \mathrm{~ms}, 38 \mathrm{~ms}$ and $46 \mathrm{~ms}$ in Figs. 7-8), one can define a control volume delimited by the chamber backplane, the combustor exit, the two quartz walls and these two vertical planes. Following the same procedure used to derive $S_{a, r e s}$, an absolute turbulent flame speed $S_{a, t}$ corresponding to the flame modeled as two vertical planes is defined:

$$
S_{a, t}=\frac{1}{A_{0}} \frac{d V_{b}}{d t}
$$

where $A_{0}$ is the area of the vertical plane perpendicular to the azimuthal direction (i.e. $A_{0}=2 h \Delta r$, where $h$ is the height of the combustion chamber and $\Delta r$ is the width of the chamber). Philip [58] demonstrated that the absolute turbulent velocity gives a relevant estimation of the propagation velocity associated to lightround time in the case of gaseous fuel. Figure 15 shows the impact of the $\beta$-formulation on $S_{a, t}$. The over-imposed gray box corresponds to phases I-II of the two calculations, where the flame cannot be modelled as two vertical planes and therefore Eq. (10) is not valid. Once the flame reaches the exit of the combustion chamber (i.e. with the beginning of phase-III), in both calculations $S_{a, t}$ shows a sudden drop, before settling to a constant value. This period, which is highlighted in the figure by horizontal arrows, corresponds to the propagation of the flame fronts as vertical planes. The plateau values of $S_{a, t}$, pointed out in Fig. 15, are $S_{a, t}^{\beta=\beta_{\text {Charlette }}}=12 \mathrm{~m} . \mathrm{s}^{-1}$ and $S_{a, t}^{\beta=\beta_{\text {dynamic }}}=9.6 \mathrm{~m} . \mathrm{s}^{-1}$, with a reduction of about $20 \%$ in the dynamic calculation. Then, advancing within phase-III, when the two fronts are approaching each other, both curves show a progressive reduction up to arriving to the merging and then to the full-ignition of the chamber.

The dynamic closure has therefore an effect on the flame propagation speed. This has been demonstrated starting from the decrease in the heat release rate that is followed by a drop in the absolute flame speeds both on the resolved flame front and from a global point of view.

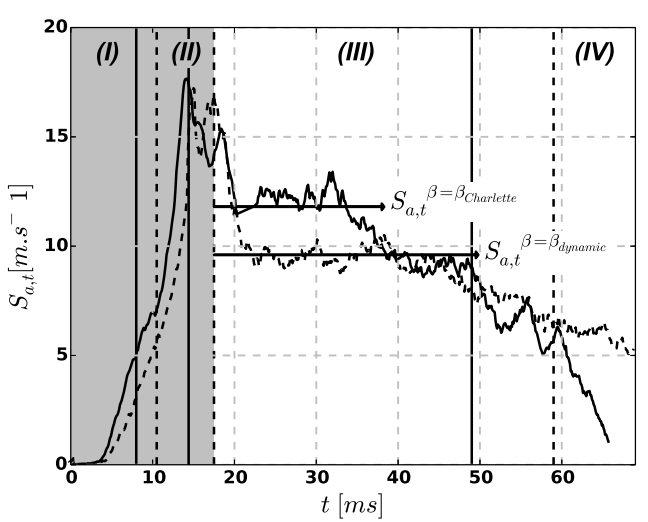

Figure 15: Time evolution of absolute turbulent flame speed computed by Eq. (10) with constant $\beta$ (solid black line) and with the dynamic formulation (dashed black line). Vertical lines highlight the beginning and the end of the numerical phases of light-round, identified by Roman numbers, for the constant (solid black line) and dynamic (dashed black line) computations.

Ultimately, to have a quantitative estimation about this point, the ignition delay time of each sector is analysed. As explained in [19], the measurements from PM give information about the time evolution of the $\mathrm{OH}^{*}$ light emissions for each sector. The time-frame corresponding to the maximum of the emission is measured as the sector ignition time and, then, the delay between consecutive sectors can be directly computed.

Figure 16 reports the ignition delay times between consecutive burners, as obtained with constant and dynamic $\beta$ formulations and compared with the experiments. Two set of data are shown for measurements (i.e. circle symbols in Fig. 16) to highlight the variability in the experimental data. In the simulations, consistently with [19], the position of the flame leading point has been used to derive the flame passage time. Instants when the LP crosses the circumferential position of each injector are plotted as solid line for the case $\beta=\beta_{\text {Charlette }}$ (thick solid line for $\mathrm{H}^{+}$and thin solid line for $\mathrm{H}^{-}$) and dashed curve for the dynamic calculation (thick dashed line for $\mathrm{H}^{+}$and thin dashed line for $\mathrm{H}^{-}$). All the cases have been synchronized based on the ignition of the first injector (i.e. $S_{1}$ or $S_{-1}$ in Fig. 1), which sets the time-origin. First, it should be pointed out that a consistent gap exists between the experiments and the case $\beta=\beta_{\text {Charlette }}$. The calculation overestimates the ignition delay between sectors, leading to a gradually increasing error in the ignition times, which entails a larger difference in the light-round duration. Finally, the discrepancy between the computation with $\beta=\beta_{\text {Charlette }}$ and 


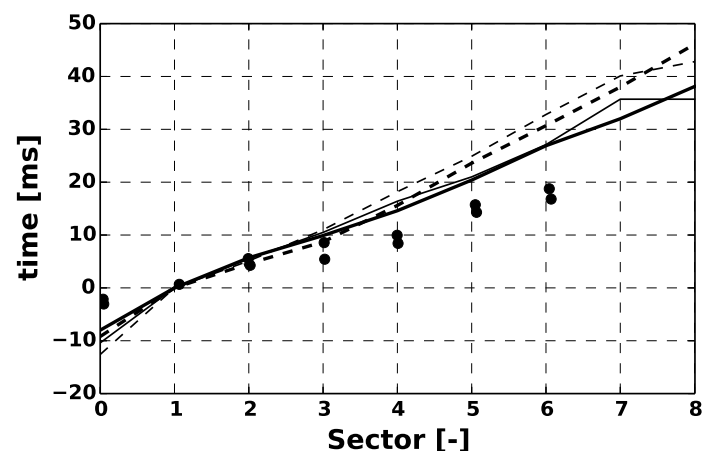

Figure 16: Ignition delay times for each sector in the experiments (circles) and in the simulations (lines). Numerical data extracted from the leading point for the constant $\beta$ case in $H^{+}$(thick solid lines) and in $H^{-}$(thin solid lines) and extracted from the dynamic calculation in $H^{+}$(thick dashed lines) and in $H^{-}$(thin dashed lines).

the experiments, in terms of light-round time, is about $34.5 \%$. The mono-disperse simplification employed to describe the liquid phase can be an important source of uncertainty. Furthermore, all the presented calculations have been carried out by assuming adiabatic walls. Indeed, in the experiments, the walls were pre-heated at a temperature corresponding to the steady-state stabilized flame regime before triggering the ignition in order to minimize heat losses in burnt gases. However, in this manner, the pre-heating of fresh gases before igniting, which can modify the flame-propagation speed, is not accounted for. Several developments are actually in progress on these modelling aspects to improve the accuracy of the employed numerical setup with respect to Fig. 16.

Nevertheless, the present work is mainly aimed at assessing the effect of the dynamic formulation on lightround. Figure 16 confirms that this has an appreciable impact. On the last injector $\left(S_{8}\right.$ in Fig. 1$)$, the ignition delay time increases by about $20 \%$ and $15 \%$ respectively for $\mathrm{H}^{+}$and $\mathrm{H}^{-}$, thus entailing a non-negligible contribution of the dynamic procedure. The same effect can be also computed by looking directly to the lightround duration $\left(\tau_{l r}\right)$. In previous works on MICCASpray $[18,19], \tau_{l r}$ has been defined as the delay between the ignition of the $S_{1}$ sector and the head-on collision of the flame fronts at the opposite side of the chamber (i.e. sector $S_{8}$ in Fig. 1). Using this definition, it gives $\tau_{l r}^{\beta=\beta_{\text {Charlete }}}=36.5 \mathrm{~ms}$ and $\tau_{l r}^{\beta=\beta_{\text {dynamic }}}=43.8 \mathrm{~ms}$. Thus, this confirms a global effect of the dynamic closure on light-round time of about the $20 \%$. This effect is expected to be independent of the formalism applied on the liquid-phase or of the inclusion of wall heat-losses and, therefore, it has to be taken into account in future light-round computations.

It should be pointed out that the impact of the dynamic procedure on $\tau_{l r}$ is consistent with the one evaluated above from $S_{a, t}$ (see Fig. 15). This aspect points out the consistency of the proposed global analysis and the relevance of the absolute turbulent speed in investigating the light-round process.

In the next section, the elements that compose $S_{a, t}$ are further deepened to point out in detail where the dynamic closure is acting during the flame propagation.

\subsection{Impact of $\beta$ on the wrinkling factor}

The global effect of the dynamic closure shown in Figs 15-16 can be studied to point out where it comes from and which are the main flame characteristics that are modified by changing the flame wrinkling parameter. In particular, the proposed analysis is based on splitting $S_{a, t}$ into two main contributions, i.e. one associated to the turbulent flame consumption speed and one linked to the evacuation of burnt gases at the outlet of the domain.

Integrating the progress variable transport equation over the combustion chamber, a macroscopic balance equation for burnt gases inside the combustor can be derived:

$$
\frac{d m_{b}}{d t}+\dot{m}_{b}^{\text {out }}-\dot{m}_{b}^{\text {in }}=\int_{V_{c c}} \overline{\dot{\omega}_{c}} d V,
$$

where $m_{b}$ is the mass of burnt gases, $\dot{m}_{b}{ }^{\text {out }}$ is the burnt gases mass flow rate at the combustor outlet and $\dot{\omega}_{c}$ the progress variable reaction term. The value of the burnt gases mass flow rate at the inlet $\dot{m}_{b}^{\text {in }}$ is actually zero. Equation (11) gives an expression for the rate of variation of $V_{b}$ since $m_{b}=\rho_{b} V_{b}$, where $\rho_{b}$ is the density of burnt gases. Hence, this equation can be introduced in Eq. (10) and, neglecting the time-variation of the density of burnt gases, one can write for $S_{a, t}$ :

$$
S_{a, t}=\frac{\int_{V_{c c}} \overline{\dot{\omega}_{c}} d V}{\rho_{b} A_{0}}-\frac{\dot{m}_{b}^{\text {out }}}{\rho_{b} A_{0}}
$$

The absolute flame speed is then related to the turbulent flame consumption velocity defined as:

$$
S_{c, t}=\frac{\int_{V_{c c}} \overline{\dot{\omega}_{c}} d V}{\rho_{u} A_{0}}
$$

where $\rho_{u}$ is the density in unburnt gases, assumed as uniform. The relationship between both velocities, 
defined during phase-III by assimilating the turbulent flame to two vertical planes, is therefore:

$$
S_{a, t}=\underbrace{\frac{\rho_{u}}{\rho_{b}} S_{c}{ }^{t}}_{\text {Term A }}-\underbrace{\frac{\dot{m}_{b}^{\text {out }}}{\rho_{b} A_{0}}}_{\text {Term B }}
$$

Based on Eq. (14), two limiting cases for $S_{a, t}$ can be recognized: in steady state conditions, the absolute speed of the flame is zero, and therefore the total reaction rate is related to the total outlet mass flow rate. In a confined system instead ( $\dot{m}_{b}^{\text {out }}=0$ ), the classical expression $S_{a, t}=\frac{\rho_{u}}{\rho_{b}} S_{c, t}$ is retrieved: the effect of the burnt gases expansion and the corresponding induced flow that transports and accelerates the flame make the ratio of density appear. Hence, the outlet flow rate of burnt gases accounts for the transition between these two limiting regimes as ignition proceeds in a semi-confined configuration as MICCA-Spray.

The two parts that compose the absolute turbulent flame speed are plotted in Fig. 17 and are analysed hereinafter:

- Term A: This contribution accounts for the component of the turbulent absolute flame speed associated to turbulent combustion only. It is shown in Fig. 17(a) (only phase-III is discussed). Consistently with the evolution of the integrated heat release shown in Fig. 13, both constant and dynamic $\beta$ curves in Fig. 17(a) evolve with a rather constant slope. The dynamic formulation shows values smaller than in the other case, thus indicating a slower flame. Such difference is associated to the different formalism on $\beta$ and to the induced drop in the wrinkling parameter. Indeed, starting from the definition of $S_{c, t}$, it is possible to demonstrate that:

$$
S_{c, t}=\frac{\int_{V_{c c}} \overline{\dot{\omega}_{c}} d V}{\rho_{u} A_{0}}=\frac{S_{c}{ }^{\Delta} A_{r e s}}{A_{0}}
$$

where $S_{c}{ }^{\Delta}$ is the mean consumption speed of the resolved flame front, which can be computed as:

$$
S_{c}{ }^{\Delta}=\frac{1}{\rho_{u} A_{r e s}} \int_{V_{c c}} \overline{\dot{\omega}_{c}} d V
$$

In a similar fashion, the consumption speed for a flame front fully resolved (i.e. within a Direct Numerical Simulation framework) in a flamelet regime and with a moderate effect of the curvature can be computed as:

$$
S_{c}=\frac{1}{\rho_{u} A_{t o t}} \int_{V_{c c}} \dot{\omega}_{c} d V
$$

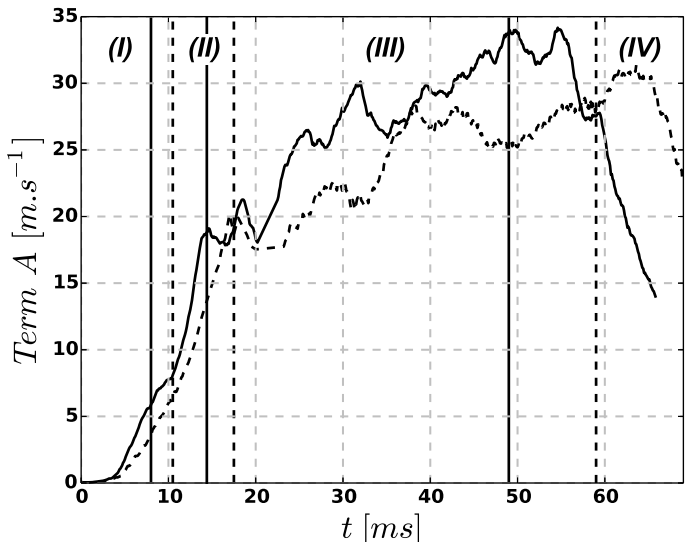

(a)

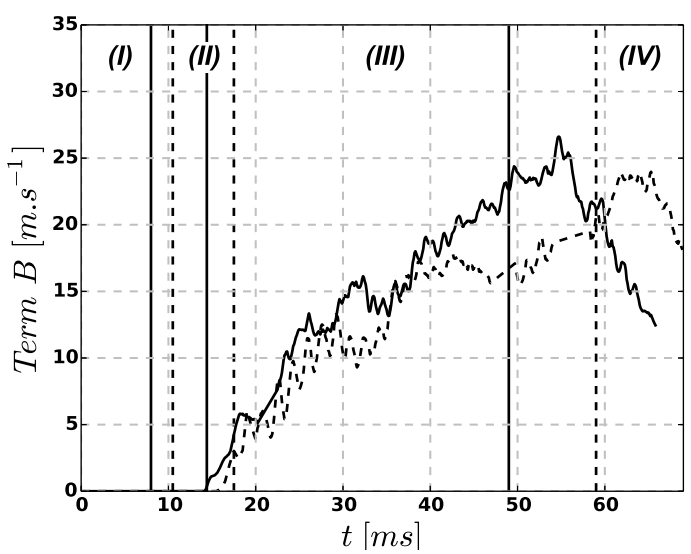

(b)

Figure 17: Contributions to the absolute turbulent flame speed with constant and dynamic formulations: Term A on the left-side and Term $B$ on the right-side. Solid black line represents the constant $\beta$ case, whereas dashed black line shows the results with the dynamic formulation. Vertical lines highlight the beginning and the end of the numerical phases of light-round, identified by Roman numbers, for the constant (solid black line) and dynamic (dashed black line) computations.

where $A_{t o t}=\int_{V_{c c}} \Xi_{\Delta}|\nabla \tilde{c}| d V$ is the total flame surface. Combining Eqs. (16)-(17), it is possible to recast the following expression for $S_{c}{ }^{\Delta}$.

$$
S_{c}{ }^{\Delta}=S_{l} \frac{A_{\text {tot }}}{A_{\text {res }}}=S_{l}\left\{\Xi_{\Delta}\right\}
$$

where $\left\{\Xi_{\Delta}\right\}$ represents the averaged value of the subgrid-scale wrinkling factor over the resolved flame front. Finally, $S_{c, t}$ can be expressed as:

$$
S_{c, t}=\frac{S_{l}\left\{\Xi_{\Delta}\right\} A_{\text {res }}}{A_{0}}=S_{l}\left\{\Xi_{\Delta}\right\} \Xi_{r e s}
$$


where $\Xi_{\text {res }}=A_{\text {res }} / A_{0}$ is the resolved flame wrinkling. Then, considering that between constant and dynamic computations the same value of $S_{l}$ is prescribed as an input, the gap in $S_{c, t}$, shown in Fig. 17(a), can be associated to a different prediction of $\Xi_{\text {res }}$ or $\left\{\Xi_{\Delta}\right\}$. These two quantities are displayed in Fig. 18 for the two calculations. The

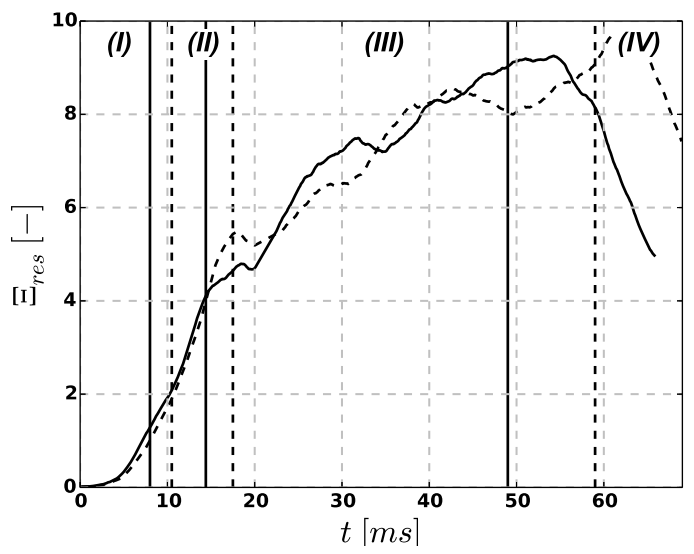

(a)

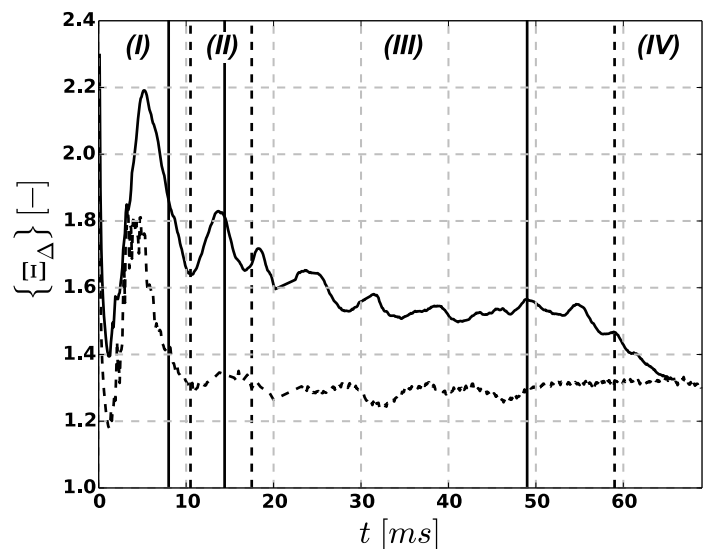

(b)

Figure 18: Time evolution of the resolved wrinkling $\left(\Xi_{\text {res }}\right)$ (a) and of the averaged value of the subgrid-scale wrinkling over the resolved flame front $\left(\left\{\Xi_{\Delta}\right\}\right)(\mathrm{b})$. Solid black line represents the constant $\beta$ case, whereas dashed black line shows the results with the dynamic formulation.

two plots clearly show that the difference in $S_{c, t}$ is linked to a reduction of $\left\{\Xi_{\Delta}\right\}$ in the dynamic case. As expected, reducing $\beta$ and entering it in Eq. (1), the wrinkling factor $\left\{\Xi_{\Delta}\right\}$ is lowered and consequently the flame propagation speed. Instead, the impact of the dynamic formulation on $A_{r e s}$ is mod- erate and it is not the key to justify the difference shown in Fig. 17(a).

- Term B: This part of $S_{a, t}$ accounts for the evacuation of burnt gases at the outlet of the combustion chamber. It is shown in Fig. 17(b). Initially, it is zero since the flame is propagating within the sector $S_{0}$ and then it increases up to reaching a maximum once the combustion chamber has been fully ignited. The evolution in the two investigated cases is very similar for large part of the lightround, even if the slope with the dynamic formulation is lower. Nevertheless, the gap between the two curves is small until $t=40 \mathrm{~ms}$, where the two simulations start showing different time evolution. However, it should be pointed out that in the calculation with $\beta=\beta_{\text {Charlette }}$ at this time the two flame fronts are approaching the merging, whereas with the dynamic formulation they are still propagating in the form of two vertical planes. Therefore, if the comparison is just focused in the time-span where both computations are in phase-III, the difference in $S_{b, \text { out }}$ is small and it cannot justify the gap in $S_{a, t}$ pointed out in the previous section.

Hence, this analysis suggests that the difference in $S_{a, t}$, detected in the dynamic case, is associated to the drop in $S_{c, t}$, which is related to the modification of the wrinkling factor. This decrease, in turn, is not balanced by an increase of the resolved flame surface. Therefore, the augmentation in the light-round and ignition delay times by $20 \%$ with dynamic $\beta$ is linked to the $S_{a, t}$ contribution connected to the turbulent consumption speed. This component is directly affected by the drop in the wrinkling parameter.

Finally, it is worth mentioning that, albeit the global effect of the dynamic closure is noticeable, its local impact on the wrinkling factor is even higher.

Figure 19 shows the time-evolution of $\Xi_{\Delta}$ on the flame leading point in the two halves of the chamber in the constant and dynamic calculations. The local variation in time of the wrinkling factor can be much larger than what was retrieved from a global point of view. The difference is quite large during the initial phase of the flame propagation and tends to diminish at the end of light-round once the LP is reaching the opposite side of the chamber. Clearly, this comparison suffers from the possible differences that can arise in Eq. (1) for the variations in $u_{\Delta}^{\prime}, R e_{\Delta}{ }^{\prime}$ or equivalence ratio (i.e. $\left.\delta_{l}, S_{l}\right)$ in the two computations.

However, this result supports the idea that the impact of the wrinkling parameter investigated in the previous section from a global perspective is the result of more 


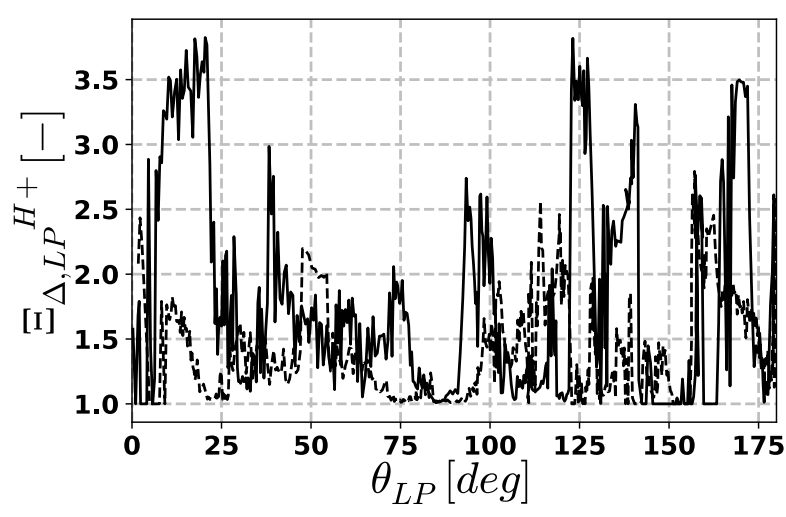

(a)

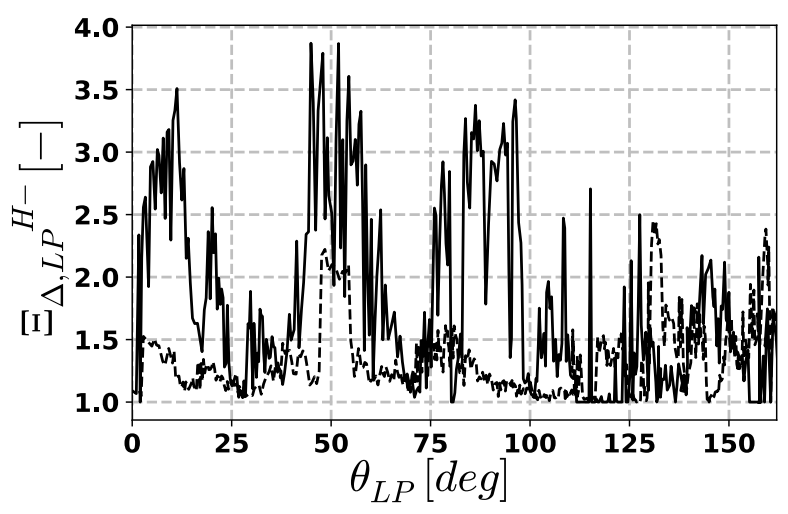

(b)

Figure 19: Time evolution of the subgrid-scale wrinkling $\Xi_{\Delta}$ at the flame leading point between constant (continuous black line) and dynamic (dashed black line) $\beta$ calculations for the two halves of the chamber $\left(H^{+}\right.$-halve (a) and $H^{-}$-halve (b)).

significant local modifications in $\Xi_{\Delta}$. This observation enforces the importance of dynamic modelling in lightround computations and the need to take it into account to have a consistent representation of the flame propagation mechanisms from global and local perspectives.

\section{Conclusions}

This article presents numerical results on the lightround process in a liquid-fuelled annular combustion chamber and focuses on the impact of the flame subgridscale wrinkling modelling. The effects of a dynamic subgrid formulation on aero-engines light-round have not been documented previously. Two formulations for the wrinkling factor have been compared: the first one proposed by Charlette et al. [52] and the other based on a dynamic calculation of the wrinkling parameter $\beta$ entering the subgrid flame wrinkling.

Numerical calculations allowed a detailed comparison between the two strategies and the following main conclusions can be summarized:

- During the light-round, the $\beta$ field presents a bimodal distribution with different behaviours in the flame-propagation region and for the flames attached around each injector. A significant drop with respect to the standard value $\beta_{\text {Charlette }}=0.5$ has been reported in the regions where the flame moves from one injector to the following one. This result proves the importance of dynamic approach for this application.

- The effects of the modifications in the wrinkling parameter on the flame propagation speed were investigated. In particular, the drop in the absolute flame speed induced by dynamic modelling has been analysed by studying the two components that control $S_{a, t}$, i.e. one associated to the subgrid and resolved wrinkling and one linked to the flowrate of burnt gases at the outlet of the chamber. It has been concluded that the dynamic approach induces a reduction of the subgrid-scale wrinkling factor and therefore of the turbulent consumption speed.

- The dynamic formulation eventually leads to a $20 \%$ increase in the light-round duration showing a non-negligible effect that should be accounted for in future light-round simulations. This quantification comes from the comparison of single realisations obtained with constant and dynamic formulations. In this comparison, one should not forget the inherent stochastic nature of investigated ignition process. While multiple realisations for each modelling setup (i.e. different initial conditions) is here prohibitive, the thoroughly and consistently tracked effect of the dynamic model on the LES results gives utmost evidence to rule out this point to explain the origin of the difference between both model formulations.

Neither the strategy of assuming $\beta_{\text {Charlette }}=0.5$ nor a tuning of the wrinkling parameter would lead to a consistent representation of the wrinkling factor. The dualzone evolution of $\beta$ that has been highlighted in the present work can be just correctly represented by using a dynamic approach for the wrinkling factor. Furthermore, beyond the effect on $\tau^{l r}$, the impact of the dynamic closure on the wrinkling parameter is even more 
critical if local quantities are studied. This conclusion has been obtained for MICCA-Spray at ambient condition, but it gives a modelling suggestion that has to be retained also at altitude relight, when ignition and lightround becomes critical aspects.

Nevertheless, despite the dynamic approach is more general and it presents a consistent effect on the different elements of the computation, the result for the dynamic formulation is worse than assuming $\beta_{\text {Charlette }}=0.5$ when compared to the experimental light-round duration. Since the assessed model for the flame wrinkling factor is definitely better, cancelling errors from other modeled parts must actually be responsible for the better result of the static formulation. The present study disrupts this erroneous balance and reveals shortcomings in other aspects of the modeling setup that have not been considered so far because the static formulation's results seemed satisfactory up to now.

One the one hand, the studied physical problem could be simplified by considering a gaseous fuel to remove the impact of the two-phase flow formulation. This would however put the configuration further away from practical and industrial considerations. On the other hand, future studies should tackle other modeling improvements that have been here disregarded. A monodisperse Eulerian-Eulerian injection was employed for the liquid phase to reduce the overall computational cost. Future studies will have to consider the EulerianLagrangian approach to describe the liquid-gas interactions in polydisperse sprays, which can be an important aspect during the light-round process. Moreover, the wall heat losses were not accounted for, relying on the assumption of adiabatic walls. However, they can have a first-order impact on the flame propagation for MICCA-Spray and deserve more attention to reproduce the experimental findings properly, as shown in [59]. Ultimately, the simplified two step chemical description represents a further element to be improved. A study to investigate the effects of detailed chemistry would be interesting and advanced techniques such as ARC[50] or virtual chemistry [51] could be considered in this context.

The combination of the dynamic closure with these improvements will be pursued in the next future to obtain a better agreement with the experiments.

\section{Acknowledgments}

This work was granted access to the HPC resources of TGCC and IDRIS under the allocation 2017A0022B10118 made by GENCI and the PRACE allocation n14-2016153551. This work was supported by

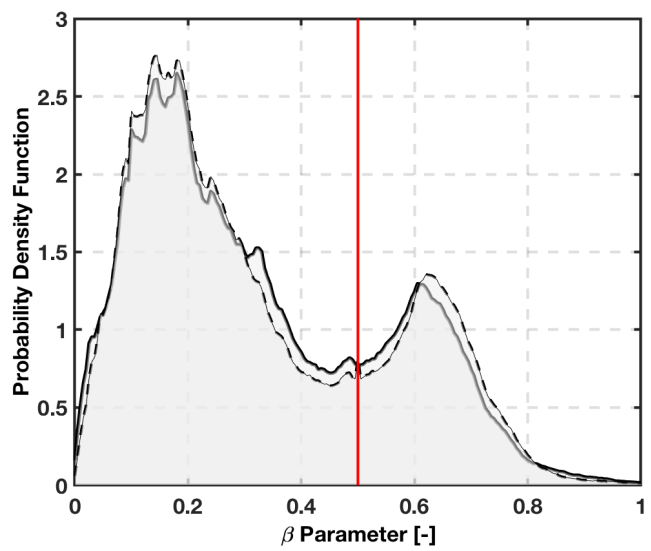

Figure 20: Comparison of the $\operatorname{PDF}(\beta)$ obtained on all the points where $\epsilon \leq \tilde{c} \leq 1-\epsilon(\epsilon=0.02)$ at $t=38 \mathrm{~ms}$ when the limitation on the filtering procedure is applied (continuous black line) and when it is removed (dashed black line).

grant ANR14-CE23-0009-01 of the French Agence Nationale de la Recherche in the framework of the TIMBER project. Dr. Olivier Colin is also gratefully acknowledged for the exchanges regarding the dynamic correction proposed in [54].

\section{Appendix A: Effect of the limitation of the filtering operation on the $\beta$ parameter}

In Sec. 3.2, it is reported that, the filtering operation has been limited to the combustion chamber (thus avoiding the plenum and the atmosphere) and to the zones where the flame is located. The clipping on the flame region has been carried out by restricting the filtering operation where the thickening factor is higher than unity. The effect of this choice has been verified using the data at $t=38 \mathrm{~ms}$ in Fig. 8. This time-frame has been selected since the flame has already reached half of the combustion chamber, so as it is possible to assess the impact of the filtering operation both for the propagating flame fronts and for the flames stabilized around each injector. To have a meaningful comparison, in this figure, the wrinkling parameter has been extracted for all points where $\epsilon \leq \tilde{c} \leq 1-\epsilon$, where $\epsilon=0.02$. The comparison between the $\operatorname{PDF}(\beta)$ obtained by applying the limitation on the filtering operation and the one extracted without any restriction is shown in Fig. 20. It should be pointed out that the two PDFs are almost overlapping with small differences, probably associated with the boundary zones where the flame is advancing. However, such discrepancies do not modify at all the main 
characteristics of the $\beta$ distribution, and therefore the discussion carried out in the present study. With this approach, the computational performances of the dynamic procedure are enhanced, arriving to the additional overcost of $20 \%$ reported in Sec. 3.2 with respect to the constant case. In [60], an over-cost of $60 \%$ due to dynamic modelling was reported. Furthermore, by comparing the $\operatorname{PDF}(\beta)$ shown in Fig. 20 with the one previously reported in Fig. 8 it should be pointed out that the shape and the modes values of the distribution of the wrinkling parameter are the same regardless of choice in terms of progress variable clipping. This observation further enforces the relevance and the general meaning of the discussion carried out in Sec. 4.1 through the iso-surface of $\tilde{c}=0.5$.

\section{Appendix B: Definition of the average absolute ve- locity over the resolved flame surface $S_{a, r e s}$}

In Sec.4.2, the absolute velocity of a flame front element has been defined as:

$$
S_{a}=\mathbf{w} \cdot \mathbf{n}=\frac{1}{|\nabla \tilde{c}|} \frac{\partial \tilde{c}}{\partial t}
$$

with $\mathbf{w}$ the absolute velocity (in a fixed referential system) of a $\tilde{c}$ iso-surface and $\mathbf{n}$ the vector normal to the flame front. All the $\tilde{c}$ iso-surfaces are supposed to have the same absolute velocity, so that $\mathbf{w}$ does not depend on progress variable. The volume of burnt gases, $V_{b}(\widetilde{c})$, in the chamber can be defined as the volume delimited by the combustor back-plane, the exit plane of the chamber, the quartz walls and the chosen iso-surface of progress variable $\mathcal{A}_{f}(\widetilde{c})$. In this manner, the variation of $V_{b}(\widetilde{c})$ in time can be written using the transport theorem and the fact that all the surfaces of the control volume except $\mathcal{A}_{f}(\widetilde{c})$ are fixed:

$$
\frac{d V_{b}(\widetilde{c})}{d t}=\frac{d}{d t} \int_{V_{b}(\widetilde{c})} 1 d V=\int_{\mathcal{A}_{f}(\widetilde{c})} S_{a} d A
$$

The integral of $d V_{b}(\widetilde{c}) / d t$ over all the $\widetilde{c}$ iso-surfaces gives then:

$$
\int_{0}^{1} \frac{d V_{b}(\widetilde{c})}{d t} d \widetilde{c}=\int_{0}^{1} \int_{\mathcal{A}_{f}(\widetilde{c})} S_{a} d A d \widetilde{c}
$$

The RHS can be further transformed using the formula for the integral of modulus of the gradient given in [61], leading to:

$$
\int_{0}^{1} \frac{d V_{b}(\widetilde{c})}{d t} d \widetilde{c}=\int_{0}^{\infty} \int_{\mathcal{A}_{f}(\bar{c})} S_{a} d A d \widetilde{c}=\int_{V_{c c}} S_{a}|\nabla c| d V
$$

The thickness of the flame is negligible compared to the dimensions of the chamber. Therefore, the volume of burnt gases can be considered independent from the progress variable (i.e. $V_{b}(\widetilde{c}) \approx V_{b}$ ). Hence, Eq. (23) can be reformulated as:

$$
\frac{d V_{b}}{d t} \approx \int_{\widetilde{c}=0}^{1} \frac{d V_{b}(\widetilde{c})}{d t}=\int_{V_{c c}} S_{a}|\nabla c| d V
$$

Hence, an expression can be derived for the average of the absolute velocity over the resolved flame surface $\left(S_{a, r e s}\right)$ :

$$
S_{a, r e s}=\frac{\int_{V_{c c}} S_{a}|\nabla c| d V}{A_{\text {res }}}=\frac{1}{A_{\text {res }}} \frac{d V_{b}}{d t}
$$

where $A_{\text {res }}$ is the resolved flame surface computed as $A_{\text {res }}=\int_{V_{c c}}|\nabla \tilde{c}| d V$.

[1] E. Mastorakos, Ignition of turbulent non-premixed flames, Progress in Energy and Combustion Science 35 (1) (2009) 5797.

[2] E. Mastorakos, Forced ignition of turbulent spray flames, Proc. Combust. Inst. 36 (2) (2017) 2367-2383.

[3] T. Marchione, S. Ahmed, E. Mastorakos, Ignition of turbulent swirling $n$-heptane spray flames using single and multiple sparks, Combustion and Flame 156 (1) (2009) 166-180.

[4] C. Letty, E. Mastorakos, A. R. Masri, M. Juddoo, W. O'Loughlin, Structure of igniting ethanol and n-heptane spray flames with and without swirl, Experimental Thermal and Fluid Science 43 (2012) 47-54.

[5] F. Collin-Bastiani, J. Marrero-Santiago, E. Riber, G. Cabot, B. Renou, B. Cuenot, A joint experimental and numerical study of ignition in a spray burner, Proceedings of the Combustion Institute 37 (4) (2019) 5047-5055.

[6] A. P. Wandel, Influence of scalar dissipation on flame success in turbulent sprays with spark ignition, Combustion and Flame 161 (10) (2014) 2579-2600.

[7] D. Barré, L. Esclapez, M. Cordier, E. Riber, B. Cuenot, G. Staffelbach, B. Renou, A. Vandel, L. Y. M. Gicquel, G. Cabot, Flame propagation in aeronautical swirled multiburners: Experimental and numerical investigation, Combust. Flame 161 (9) (2014) 2387-2405.

[8] J. Marrero-Santiago, A. Verdier, C. Brunet, A. Vandel, G. Godart, G. Cabot, M. Boukhalfa, B. Renou, Experimental Study of Aeronautical Ignition in a Swirled Confined Jet-Spray Burner, J. Eng. Gas Turbines Power 140 (GTP-17-1316).

[9] J. Bourgouin, D. Durox, T. Schuller, J. Beaunier, S. Candel, Ignition dynamics of an annular combustor equipped with multiple swirling injectors, Combust. Flame 160 (8) (2013) 1398-1413.

[10] K. Prieur, D. Durox, J. Beaunier, T. Schuller, S. Candel, Ignition dynamics in an annular combustor for liquid spray and premixed gaseous injection, Proc. Combust. Inst. 36 (3) (2017) 3717-3724.

[11] K. Prieur, G. Vignat, D. Durox, T. Schuller, S. Candel, Flame and Spray Dynamics During the Light-Round Process in an Annular System Equipped With Multiple Swirl Spray Injectors, Journal of Engineering for Gas Turbines and Power 141 (6).

[12] E. Machover, E. Mastorakos, Spark ignition of annular nonpremixed combustors, Experimental Thermal and Fluid Science 73 (2016) 64-70. 
[13] E. Machover, E. Mastorakos, Experimental investigation on spark ignition of annular premixed combustors, Combustion and Flame 178 (2017) 148-157.

[14] D. Zhao, Y. Xia, H. Ge, Q. Lin, G. Wang, Large Eddy Simulation of flame propagation during the ignition process in an annular multiple-injector combustor, Fuel 263 (2020) 116402.

[15] M. Boileau, G. Staffelbach, B. Cuenot, T. Poinsot, C. Bérat, LES of an ignition sequence in a gas turbine engine, Combust. Flame 154 (1) (2008) 2-22.

[16] M. Philip, M. Boileau, R. Vicquelin, T. Schmitt, D. Durox, J. Bourgouin, S. Candel, Simulation of the Ignition Process in an Annular Multiple-Injector Combustor and Comparison With Experiments, J. Eng. Gas Turbines Power 137 (GTP-14-1375).

[17] M. Philip, M. Boileau, R. Vicquelin, E. Riber, T. Schmitt, B. Cuenot, D. Durox, S. Candel, Large Eddy Simulations of the ignition sequence of an annular multiple-injector combustor, Proc. Combust. Inst. 35 (3) (2015) 3159-3166.

[18] T. Lancien, K. Prieur, D. Durox, S. Candel, R. Vicquelin, Large Eddy Simulation of Light- Round in an Annular Combustor With Liquid Spray Injection and Comparison With Experiments, J. Eng. Gas Turbines Power 137 (GTP-17-1328).

[19] T. Lancien, K. Prieur, D. Durox, S. Candel, R. Vicquelin, Leading point behavior during the ignition of an annular combustor with liquid n-heptane injectors, Proc. Combust. Inst. 37 (4) (2019) 5021-5029.

[20] Y. Xia, C. Linghu, Y. Zheng, C. Ye, C. Ma, H. Ge, G. Wang, Experimental Investigation of the Flame Front Propagation Characteristic During Light-round Ignition in an Annular Combustor, Flow, Turbulence and Combustion 103 (2019) 247-269.

[21] P. Volpiani, T. Schmitt, D. Veynante, A-posteriori tests of a dynamic thickened flame model for Large Eddy Simulations of turbulent premixed combustion, Combustion and Flame 174 (2016) 166-178.

[22] M. Germano, U. Piomelli, P. Moin, W. H. Cabot, A dynamic subgrid-scale eddy viscosity model, Physics of Fluids A: Fluid Dynamics 3 (7) (1991) 1760-1765.

[23] H. G. Im, T. S. Lund, J. H. Ferziger, Large Eddy Simulation of turbulent front propagation with dynamic subgrid models, Physics of Fluids 9 (12) (1997) 3826-3833.

[24] E. Knudsen, H. Kolla, E. R. Hawkes, H. Pitsch, LES of a premixed jet flame DNS using a strained flamelet model, Combustion and Flame 160 (12) (2013) 2911-2927.

[25] Y.-C. Chen, N. Peters, G. Schneemann, N. Wruck, U. Renz, M. S. Mansour, The detailed flame structure of highly stretched turbulent premixed methane-air flames, Combustion and Flame 107 (3) (1996) 223 - 244

[26] G. Wang, M. Boileau, D. Veynante, Implementation of a dynamic thickened flame model for large eddy simulations of turbulent premixed combustion, Combustion and Flame 158 (11) (2011) 2199-2213.

[27] P. S. Volpiani, T. Schmitt, D. Veynante, Large Eddy Simulation of a turbulent swirling premixed flame coupling the TFLES model with a dynamic wrinkling formulation, Combustion and Flame 180 (2017) 124-135.

[28] W. Meier, P. Weigand, X. Duan, R. Giezendanner-Thoben, Detailed characterization of the dynamics of thermoacoustic pulsations in a lean premixed swirl flame, Combustion and Flame 150 (1) (2007) 2-26.

[29] C. Dem, M. Stöhr, C. Arndt, A. Steinberg, W. Meier, Experimental study of turbulence-chemistry interactions in perfectly and partially premixed confined swirl flames, Zeitschrift fur Physikalische Chemie 229 (4) (2015) 569-585.

[30] M. Boger, D. Veynante, H. Boughanem, A. Trouvé, Direct numerical simulation analysis of flame surface density concept for Large Eddy Simulation of turbulent premixed combustion, Sym- posium (International) on Combustion 27 (1) (1998) 917-925.

[31] G. Wang, M. Boileau, D. Veynante, K. Truffin, Large Eddy Simulation of a growing turbulent premixed flame kernel using a dynamic flame surface density model, Combustion and Flame 159 (8) (2012) 2742-2754.

[32] T. Schmitt, A. Sadiki, B. Fiorina, D. Veynante, Impact of dynamic wrinkling model on the prediction accuracy using the F-TACLES combustion model in swirling premixed turbulent flames, Proceedings of the Combustion Institute 34 (1) (2013) 1261-1268.

[33] R. Mercier, T. Schmitt, D. Veynante, B. Fiorina, The influence of combustion SGS submodels on the resolved flame propagation. Application to the LES of the Cambridge stratified flames, Proceedings of the Combustion Institute 35 (2) (2015) 12591267.

[34] C. Schneider, A. Dreizler, J. Janicka, Fluid Dynamical Analysis of Atmospheric Reacting and Isothermal Swirling Flows, Flow, Turbulence and Combustion 74 (1) (2005) 103-127.

[35] M. Sweeney, S. Hochgreb, R. Barlow, The structure of premixed and stratified low turbulence flames, Combustion and Flame 158 (5) (2011) 935-948.

[36] S. Ibrahim, S. Gubba, A. Masri, W. Malalasekera, Calculations of explosion deflagrating flames using a dynamic flame surface density model, Journal of Loss Prevention in the Process Industries 22 (3) (2009) 258-264.

[37] S. R. Gubba, S. S. Ibrahim, W. Malalasekera, Dynamic flame surface density modelling of flame deflagration in vented explosion, Combustion, Explosion, and Shock Waves 48 (4) (2012) 393-405.

[38] R. Knikker, D. Veynante, C. Meneveau, A priori testing of a similarity model for Large Eddy Simulations of turbulent premixed combustion, Proceedings of the Combustion Institute 29 (2) (2002) 2105-2111.

[39] P. S. Volpiani, T. Schmitt, O. Vermorel, P. Quillatre, D. Veynante, Large Eddy simulation of explosion deflagrating flames using a dynamic wrinkling formulation, Combustion and Flame 186 (2017) 17-31.

[40] A. R. Masri, A. AlHarbi, S. Meares, S. S. Ibrahim, A Comparative Study of Turbulent Premixed Flames Propagating Past Repeated Obstacles, Industrial \& Engineering Chemistry Research 51 (22) (2012) 7690-7703.

[41] K. Prieur, Dynamique de la combustion dans un foyer annulaire multi-injecteurs diphasique, Ph.D. thesis, Universite ParisSaclay, 2017.

[42] V. Moureau, G. Lartigue, Y. Sommerer, C. Angelberger, O. Colin, T. Poinsot, Numerical methods for unsteady compressible multi-component reacting flows on fixed and moving grids, J. Comput. Phys. 202 (2) (2005) 710-736.

[43] F. Nicoud, F. Ducros, Subgrid-scale stress modelling based on the square of the velocity gradient tensor, Flow Turbul. Combust. 62 (3) (1999) 183-200.

[44] M. Boileau, S. Pascaud, E. Riber, B. Cuenot, L. Y. M. Gicquel, T. Poinsot, M. Cazalens, Investigation of Two-Fluid Methods for Large Eddy Simulation of Spray Combustion in Gas Turbines, Flow Turbul. Combust. 80 (3) (2008) 291-321.

[45] B. Abramzon, W. Sirignano, Droplet vaporization model for spray combustion calculations, Int. J. Heat Mass Transfer 32 (9) (1989) 1605-1618.

[46] O. Colin, M. Rudgyard, Development of High-Order TaylorGalerkin Schemes for LES, J. Comput. Phys. 162 (2) (2000) 338-371.

[47] O. Colin, F. Ducros, D. Veynante, T. Poinsot, A thickened flame model for Large Eddy Simulations of turbulent premixed combustion, Phys. Fluids 12 (7) (1977) 1503-1515.

[48] T. Lancien, Étude numérique de l'allumage diphasique de foyers 
annulaires multi-brûleurs, Ph.D. thesis, Université Paris-Saclay, 2018.

[49] F. Shum-Kivan, J. M. Santiago, A. Verdier, E. Riber, B. Renou, G. Cabot, B. Cuenot, Experimental and numerical analysis of a turbulent spray flame structure, Proc. Combust. Inst. 36 (2) (2017) 2567-2575.

[50] A. Felden, P. Pepiot, L. Esclapez, E. Riber, B. Cuenot, Including analytically reduced chemistry (ARC) in CFD applications, Acta Astronautica 158 (2019) 444-459.

[51] M. Cailler, N. Darabiha, B. Fiorina, Development of a virtual optimized chemistry method. Application to hydrocarbon/air combustion, Combustion and Flame 211 (2020) 281-302.

[52] F. Charlette, C. Meneveau, D. Veynante, A power-law flame wrinkling model for LES of premixed turbulent combustion Part I: non-dynamic formulation and initial tests, Combust. Flame 131 (1) (2002) 159-180.

[53] S. Richard, O. Colin, O. Vermorel, A. Benkenida, C. Angelberger, D. Veynante, Towards Large Eddy Simulation of combustion in spark ignition engines, Proceedings of the Combustion Institute 31 (2) (2007) 3059-3066.

[54] S. Mouriaux, O. Colin, D. Veynante, Adaptation of a dynamic wrinkling model to an engine configuration, Proceedings of the Combustion Institute 36 (3) (2017) 3415-3422.

[55] D. Veynante, V. Moureau, Analysis of dynamic models for Large Eddy Simulations of turbulent premixed combustion, Combustion and Flame 162 (12) (2015) 4622-4642.

[56] F. Charlette, C. Meneveau, D. Veynante, A power-law flame wrinkling model for LES of premixed turbulent combustion Part II: dynamic formulation, Combustion and Flame 131 (12) (2002) 181-197.

[57] T. Poinsot, S. Lelef, Boundary conditions for direct simulations of compressible viscous flows, J. Comput. Phys. 101 (1) (1992) 104-129.

[58] M. Philip, Dynamique de l'allumage circulaire dans les foyers annulaires multi-injecteurs, Ph.D. thesis, Université ParisSaclay, 2016.

[59] S. Puggelli, T. Lancien, K. Prieur, D. Durox, S. Candel, R. Vicquelin, Impact of Wall Temperature in Large Eddy Simulation of Light-Round in an Annular Liquid Fueled Combustor and Assessment of Wall Models, Journal of Engineering for Gas Turbines and Power 142 (1).

[60] B. Rochette, F. Collin-Bastiani, L. Gicquel, O. Vermorel, D. Veynante, T. Poinsot, Influence of chemical schemes, numerical method and dynamic turbulent combustion modeling on LES of premixed turbulent flames, Combustion and Flame 191 (2018) 417-430.

[61] V. G. Maz'ja, Sobolev Spaces, Springer Series in Soviet Mathematics, 1985 